\title{
Magnetotransport signatures of chiral magnetic anomaly in the half-Heusler phase ScPtBi
}

\author{
Orest Pavlosiuk, ${ }^{1}$ Andrzej Jezierski, ${ }^{2}$ Dariusz Kaczorowski, ${ }^{1}$ and Piotr Wiśniewski ${ }^{1, *}$ \\ ${ }^{1}$ Institute of Low Temperature and Structure Research, \\ Polish Academy of Sciences, ul. Okólna 2, 50-422 Wroctaw, Poland \\ ${ }^{2}$ Institute of Molecular Physics, Polish Academy of Sciences, \\ ul. M. Smoluchowskiego 17, 60-179 Poznań, Poland
}

\begin{abstract}
Study of the magnetotransport properties of ScPtBi revealed simultaneously: a negative contribution to the longitudinal magnetoresistance, planar Hall effect, and distinct angular narrowing of the longitudinal magnetoresistance - three hallmarks of chiral magnetic anomaly (pumping of axial charge between Weyl nodes), a distinct property of topological semimetals. Electronic structure calculations show that structural defects, such as antisites and vacancies, bring a substantial density of states at the Fermi level of $\mathrm{ScPtBi}$, indicating that it is a semimetal, not a zero-gap semiconductor, as predicted earlier. This is in accord with electrical resistivity in $\mathrm{ScPtBi}$, showing no characteristics of a semiconductor. Moreover, below $0.7 \mathrm{~K}$ we observed an onset of a superconducting transition, with the resistivity disappearing completely below $0.23 \mathrm{~K}$.
\end{abstract}




\section{INTRODUCTION}

Chiral magnetic anomaly (CMA) is a unique and defining feature of topological semimetals, due to charge pumping between Weyl nodes of opposite chirality. Weyl nodes are monopoles of Berry flux and possess characteristic chiral charge (or Chern number, being integral of the Berry flux through a closed surface enclosing the node), equal to \pm 1 in a single-Weyl, or to \pm 2 in a double-Weyl semimetal ${ }^{1}$.

CMA has three main electronic magnetotransport manifestations: negative longitudinal magnetoresistance (LMR) 213, the planar Hall effect (PHE), and angular narrowing of negative LMR 415. All three have never been observed simultaneously in the same material. Negative LMR has been found in many topological semimetals, for example: TaAs, TaP, $\mathrm{ZrTe}_{5}, \mathrm{Na}_{3} \mathrm{Bi}, \mathrm{GdPtBi}$, and YbPtBi 146]10. PHE was observed less often, in $\mathrm{Cd}_{3} \mathrm{As}_{2}, \mathrm{VAl}_{3}$, $\mathrm{Na}_{3} \mathrm{Bi}$, as well as half-Heusler phases GdPtBi, YbPtBi, and DyPdBi 10 15. To date, angular narrowing of negative LMR has been found only in $\mathrm{Na}_{3} \mathrm{Bi} 4$.

ScPtBi, isostructural with GdPtBi, YbPtBi and DyPdBi, but outstanding due to yhe very light and non-magnetic scandium atom, factors important for the topological non-triviality of electronic states. It has been theoretically predicted to be a zero-gap semiconductor with inversion of electronic bands prerequisite for a $\mathbb{Z}_{2}$-type topological insulator 16]17. So far, only the observation of the weak antilocalization (WAL) effect and linear magnetoresistance suggested its topological non-triviality 18 . In this work, we synthesized the high-quality single crystals of ScPtBi and characterized them and their angle-dependent magnetotransport depending on the angle between current and applied magnetic field.

\section{METHODS}

Single crystals of ScPtBi were grown by the self-flux method from high purity constituent elements (Sc 3N, Pt 5N, Bi 6N) taken in the atomic ratio: Sc:Pt:Bi as 1:1:15, put in an

alumina crucible and sealed in a quartz ampule with argon pressure of $0.3 \mathrm{bar}$. Such a prepared ampule was quickly heated up to $1150{ }^{\circ} \mathrm{C}$ at a rate of $50{ }^{\circ} \mathrm{C} / \mathrm{h}$ and held at this temperature for 24 hours. Then it was slowly cooled down to $700{ }^{\circ} \mathrm{C}$ at a rate of $1{ }^{\circ} \mathrm{C} / \mathrm{h}$ and the excess of Bi-flux was removed by centrifugation. Obtained single crystals were pyramid-shaped and stable against air, and moisture. The largest of them had dimensions 
of $6 \times 3 \times 3 \mathrm{~mm}^{3}$ (see the right inset in Fig. S1 in the Supplemental Material ${ }^{19}$ ).

Equiatomic chemical composition and phase homogeneity of crystals were confirmed by energy-dispersive X-ray analysis using a FEI scanning electron microscope equipped with an EDAX Genesis XM4 spectrometer (Supplemental Fig. S1 19). X-ray powder diffraction experiment was carried out on powdered single crystals using an X'pert Pro (PANanalytical) diffractometer with $\mathrm{Cu}-\mathrm{K} \alpha$ radiation. Crystal structure refinement by Rietveld method was

performed with the Fullprof package 20 (a diffractogram and results of the refinement are shown in Supplemental Fig. S2A and Table S1 ${ }^{19}$ ). The results of analysis indicated that ScPtBi crystallizes within the $F \overline{4} 3 m$ space group with the lattice parameter $a=6.4412 \AA$, which differs very slightly from $a=6.50 \AA$ reported in Ref. 18. Quality and orientation of single crystals were assessed by Laue diffraction, with a LAUE-COS (Proto) system (Laue pattern is shown in Supplemental Fig. S2B 19$)$. Bar-shaped $\left(0.19 \times 0.65 \times 1.73 \mathrm{~mm}^{3}\right)$ specimen was cut from the oriented single crystal. Conventional four-probe method was used for electrical transport measurements in the temperature range from $0.05 \mathrm{~K}$ to $300 \mathrm{~K}$ and in the magnetic fields up to $14 \mathrm{~T}$, using a PPMS platform (Quantum Design) equipped with dilution refrigerator. Electrical contacts were made from $50 \mu \mathrm{m}$-thick silver wires attached to the sample with silver paint. We took special care while preparing contacts, to avoid current jetting, which could cause spurious negative LMR, as described in Refs. 13 and 15. The same PPMS platform was used for specific heat measurements.

The electronic structure was calculated by the Full-Potential Local Orbital Minimum Basis (ver. 18) method ${ }^{2122}$ in the local density approximation, with the exchange correlation potential taken in the form of Perdew, Burke, and Ernzerhof (PBE) 22123. The band structure of the ordered ScPtBi was calculated in the full-relativistic mode in general gradient approximation, including the spin-orbit interaction. For the disordered structures and for models with vacancies the band calculations were performed for the supercell of 24 atoms $\left(\mathrm{Sc}_{8} \mathrm{Pt}_{8} \mathrm{Bi}_{8}\right)$ in the full-relativistic and scalar-relativistic schemes, respectively. In the antisite models we interchanged two atoms from two different sublattices, per supercell. In the models with vacancies, one of atoms ( $\mathrm{Sc}, \mathrm{Pt}$ or $\mathrm{Bi}$ ) was vacant per supercell.

\section{RESULTS AND DISCUSSION}

Contrary to prior theoretical predictions 16 167, our ScPtBi samples exhibited a metallic-like temperature dependence of electrical resistivity, $\rho_{x x}$, shown in Fig. S4A of the Supplemental 

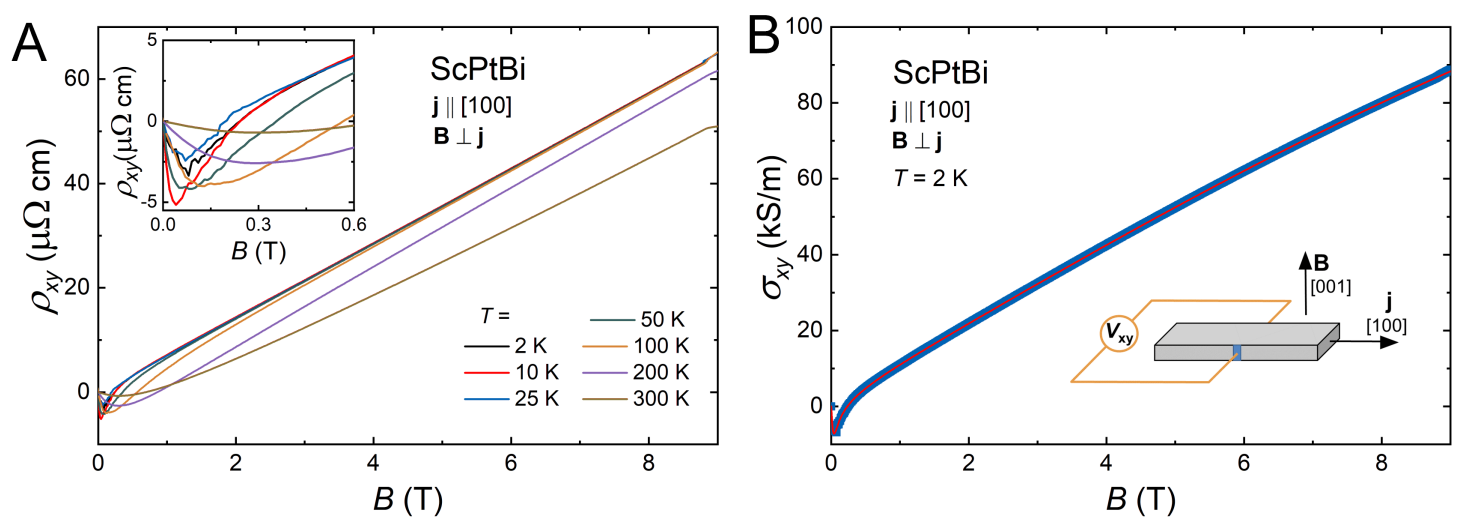

FIG. 1. (A) Hall resistivity measured at several different temperatures as a function of magnetic field with $\mathbf{j} \|[100]$ and $\mathbf{B} \|[001]$. The inset presents the Hall resistivity in weak magnetic field region. (B) Magnetic field dependent Hall conductivity measured at $T=2 \mathrm{~K}$. The red solid line represents the fit with Equation (1). Quality of the fit was excellent (with $R^{2}>99.99$ ). Inset shows the geometry of measurement.

Material 19. Moreover, zooming into the low-temperature region we observed below $0.7 \mathrm{~K}$ an onset of a superconducting transition, with $\rho_{x x}$ finally decreasing to zero below $0.23 \mathrm{~K}$ (see the inset to Fig. S4A of the Supplemental Material ${ }^{[19}$ ).

The Hall resistivity isotherms, $\rho_{x y}(B)$, are nonlinear, especially in weak magnetic fields, where they have minima, indicating two or more different bands contributing to $\rho_{x y}$ (Fig. 1 A and its inset; raw data before anti-symmetrization are shown in Supplemental Fig. S5 19 ). Previously reported rectilinear $\rho_{x y}(B)$ data for ScPtBi showed only one type of carriers contributing to the transport properties 18 , suggesting different electronic structures of our samples. Nonlinear $\rho_{x y}(B)$ have also been observed for several half-Heusler compounds: YbPtBi, LuPtBi, YPtBi, TbPdBi, and DyPdBi 15|24 27. Therefore, for a quantitative analysis of our data we used the three-band (the two-band model was tested as well, but it did not fit our data) Drude model of Hall conductivity:

$$
\sigma_{x y}(B)=\sum_{i=1}^{3} \frac{e n_{i} \mu_{i}^{2} B}{1+\left(\mu_{i} B\right)^{2}},
$$

summing up the conductivities of individual bands, with $n_{i}$ and $\mu_{i}$ denoting respectively the concentration and mobility of carriers from the $i$ th band ( $e$ is the elementary charge). We fitted Eq. S1 to the experimental Hall conductivity data calculated as $\sigma_{x y}=\rho_{x y} /\left(\rho_{x x}^{2}+\rho_{x y}^{2}\right)$, appropriate because $\rho_{x y} \ll \rho_{x x}$. The result of Eq. S1 fitting to $\sigma_{x y}(B)$ obtained at $T=2 \mathrm{~K}$ is represented by red solid line in Fig. $1 \mathrm{~B}$. The same Eq. $\mathrm{S} 1$ was fitted to the $\sigma_{x y}(B)$ data 
measured at other temperatures, the results are shown in Supplemental Fig. S6 19, and fit parameters are collected in Supplemental Table S2 19 .

Interestingly, charge mobilities derived for two of these bands are very high, especially that of an electron-like band: $\mu_{2} \approx 2 \times 10^{5} \mathrm{~cm}^{2} \mathrm{~V}^{-1} \mathrm{~s}^{-1}$, which is the highest ever observed for pnictogen-bearing half-Heusler phases, and similar to those in topological semimetals $\mathrm{ZrTe}_{5}{ }_{28}^{28}, \mathrm{TaAs}^{29}$ and $\mathrm{WTe}_{2} \stackrel{30}{\text {. }}$

Looking for topological semimetal features in ScPtBi, we measured its $\rho_{x x}$ as a function of magnetic field, $B$, and calculated the magnetoresistance, $\mathrm{MR}=\left(\rho_{x x}(B) / \rho_{x x}(0)\right)-1$, for different angles, $\theta$, between $\mathbf{B}$ and electric current, $\mathbf{j}$ (as shown in Fig. 2 A for $T=2 \mathrm{~K}$ ). $\mathrm{MR}$ is the largest in transverse configuration (TMR), i.e. with $\mathbf{B} \perp \mathbf{j}$. When $\mathbf{B}$ is rotated $\left(\theta=90^{\circ} \rightarrow 0^{\circ}\right.$, as sketched in the inset to Fig. 2A), MR decreases monotonously, down to the lowest value in an LMR configuration $\left(\theta=0^{\circ}\right)$. The field variation of LMR at $2 \mathrm{~K}$ is fairly unusual: it increases abruptly with increasing $B$, reaches a maximum of $64 \%$ in $2.75 \mathrm{~T}$, and decreases gradually in stronger fields.

The magnetic field dependence of LMR changes prominently with temperature (Fig. 2B). Negative slope of $\operatorname{LMR}(B)$ at $T=2 \mathrm{~K}$, gradually becomes positive as temperature increases. This behavior can be ascribed to the presence of at least two contributions: increasing-infield (dominating in weak fields) and decreasing-in-field (taking over in stronger fields).

Finite LMR has been observed for many compounds, and when positive, it can be as-

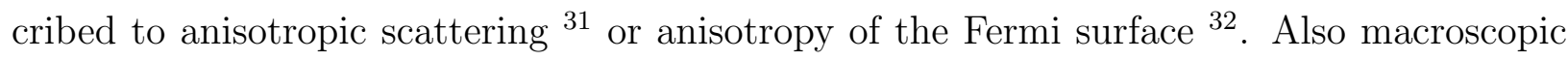

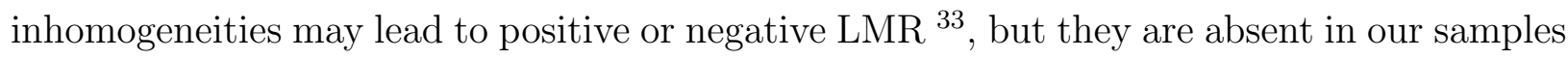
(Supplemental Fig. S1 19). LMR in topological semimetals is characterized by a distinct, additional negative contribution from CMA ${ }^{3}$. Therefore, we analyzed LMR in ScPtBi to check for its presence.

MR measured for ScPtBi in both transverse and longitudinal configuration, demonstrates very sharp increase in weak magnetic field region (Fig. 24A), that can be ascribed to WAL 18 . In stronger fields the increase of TMR becomes much slower. In order to describe this complex variation of TMR, we combined a WAL contribution with three-band Drude magnetoconductivity calculated as:

$$
\sigma_{x x}^{D}(B)=\sum_{i=1}^{3} \frac{e n_{i} \mu_{i}}{1+\left(\mu_{i} B\right)^{2}},
$$

using the concentrations and mobilities of charge carriers obtained in the analysis of Hall 

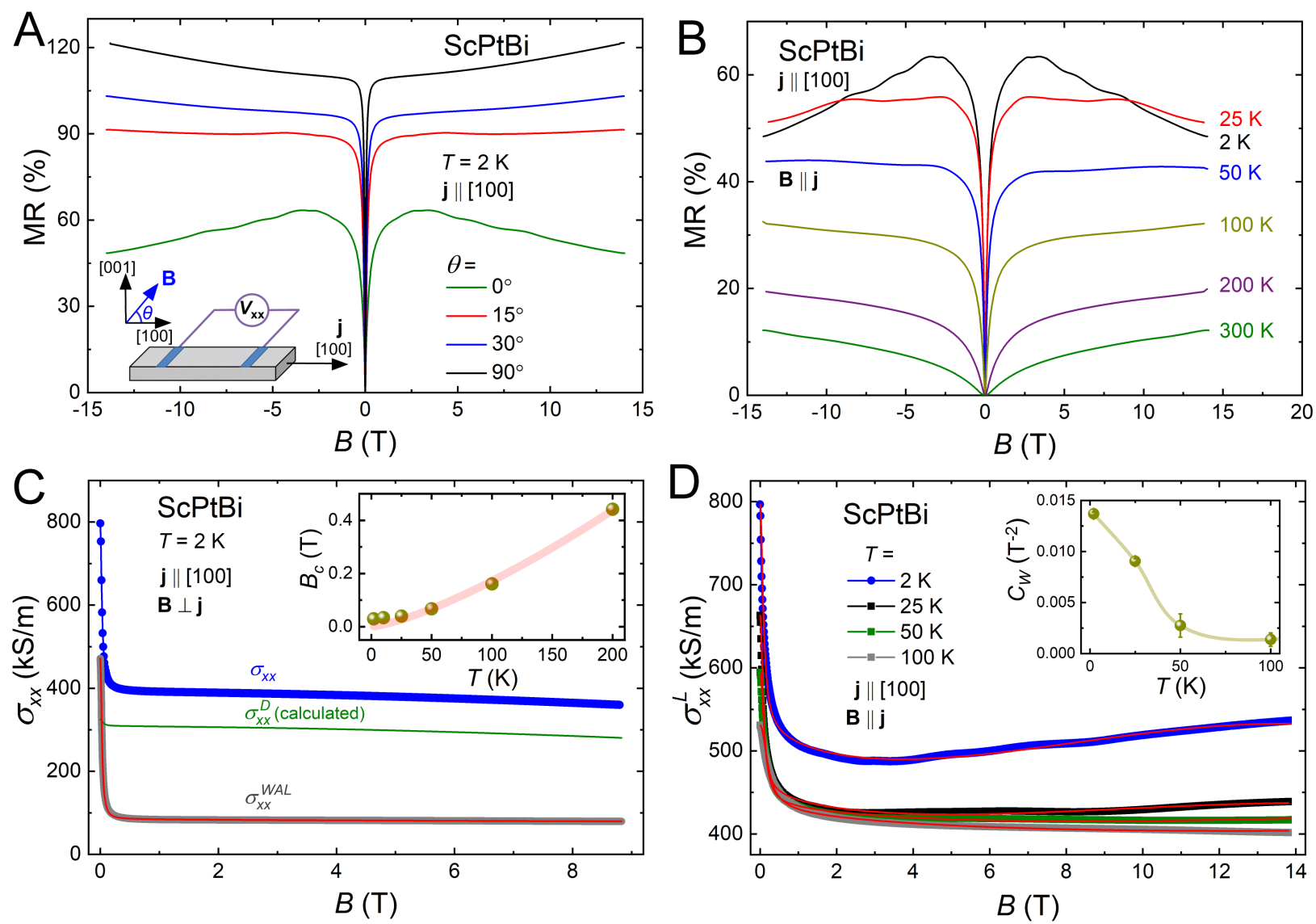

FIG. 2. (A) $\mathrm{MR}(B)$ isotherms measured at $T=2 \mathrm{~K}$ for different angles between current and magnetic field. Inset shows schematically the geometry of measurement. (B) $\operatorname{LMR}(B)$ isotherms for several temperatures. (C) Analysis of the transverse magnetoconductivity data obtained at $T=2 \mathrm{~K}$. Blue points - experimental data, green solid line - $\sigma_{x x}^{D}(B)$ calculated with Eq. (2). Grey solid curve represents the WAL contribution derived as described in the text, and red solid line curve shows the fit of Eq. (3). Inset: temperature dependence of the critical field approximated by power law $B_{c} \propto T^{1.35}$. (D) Magnetic field dependence of the longitudinal magnetoconductivity for several temperatures. Red solid lines correspond to the fits of Eq. (4). Inset: temperaturedependent chiral coefficient (solid line is a guide for the eye).

resistivity (cf. Supplemental Table $\mathrm{S} 2{ }^{19}$ ). $\sigma_{x x}^{D}(B)$ for $2 \mathrm{~K}$ is drawn in Fig. $2 \mathrm{C}$ as a green solid line. Next, we subtracted $\sigma_{x x}^{D}(B)$ from the transverse electrical magnetoconductivity $\sigma_{x x}\left[=\rho_{x x} /\left(\rho_{x x}^{2}+\rho_{x y}^{2}\right)\right]$ (blue points). In this way we obtained the WAL contribution $\sigma_{x x}^{W A L}$ to TMR in ScPtBi, shown in Fig. 2 $\mathrm{C}$ as gray solid line. Remarkably, we could very well 
approximate it with the equation proposed to describe WAL in Weyl semimetals:

$$
\sigma_{x x}^{W A L}(B)=C_{1}^{q i} \frac{B^{2} \sqrt{B}}{B_{c}^{2}+B^{2}}+C_{2}^{q i} \frac{B_{c}^{2} B^{2}}{B_{c}^{2}+B^{2}}+\sigma_{0},
$$

where $\sigma_{0}$ is the electrical conductivity in zero magnetic field, $B_{c}$ stands for the critical field related to the phase coherence length, $l_{\phi}$, as $B_{c} \sim \hbar /\left(e l_{\phi}^{2}\right) \underline{34}$. Fitting this Equation to $\sigma_{x x}^{W A L}$ data (as shown by red line in Fig. 2 C), we obtained parameters listed in Supplemental Table S3A 19 .

According to Ref. $\frac{34}{34}$ at low temperatures the low-field magnetoconductivity is proportional to $+\sqrt{B}$ in double-Weyl semimetals and to $-\sqrt{B}$ in single-Weyl semimetals. For $\sigma_{x x}$ of our sample at $T=2 \mathrm{~K}$, the critical field, $B_{c}$, is very small (0.029 $\mathrm{T}$ in Table $\left.\mathrm{S} 3\right)$, thus the first term of Eq. 3 is very well approximated with $C_{1}^{q i} \sqrt{B}$. Therefore (since $C_{1}^{q i}<0$ ) the lowfield $\sigma_{x x}$ is proportional to $-\sqrt{B}$, which allows us to conclude that ScPtBi is a single-Weyl semimetal.

Analysis of WAL we performed for TMR at higher temperatures is shown in Supplemental Fig. S7 and Table S3 19. Temperature variation of critical field $B_{c}$, shown in the inset to Fig. $2 \mathrm{C}$, can be well approximated with a power law $B_{c}=A T^{p}$, with $A=3.47 \times 10^{-4} \mathrm{~T} / \mathrm{K}$ and $p=1.35$. This value of $p$ is close to 1.5 , theoretical value for electron-electron interaction, the principal mechanism of decoherence, indicating that our estimation of the WAL contribution is adequate.

In single-Weyl semimetals WAL is isotropic ${ }^{35}$, contributing to both the longitudinal and transverse magnetoconductivity in the same way. Lorentz force acting on electrons in parallel magnetic and electric fields is zero, hence LMR is not affected by orbital magnetoresistance $\mathrm{E}^{32}$. Thus only contributions from WAL and CMA must be taken into account. We obtained the longitudinal magnetoconductivity $\sigma_{x x}^{L}(B)$ in ScPtBi as inverse of the electrical resistivity measured in longitudinal magnetic field: $\sigma_{x x}^{L}(B)=1 /\left.\rho_{x x}(B)\right|_{\mathbf{B} \| \mathbf{j}}$ (plotted in Fig. 2D). Fitting these data with the formula appropriate for Weyl semimetals 36 :

$$
\sigma_{x x}^{L}=\sigma_{x x}^{W A L}(B) \cdot\left(1+C_{W} B^{2}\right)+\sigma_{n}
$$

(represented by red solid lines in Fig. 2D) where $\sigma_{n}=\sigma_{x x}^{L}(B=0)-\sigma_{0}$, gave the parameters gathered in Supplemental Table S3B 19 . This analysis reveals that the negative contribution to LMR that we observed may be due to CMA. It weakens with increasing temperature, as reflected by the decrease of the chiral coefficient, $C_{W}$ (cf. the inset to Fig. 2D). The chiral 

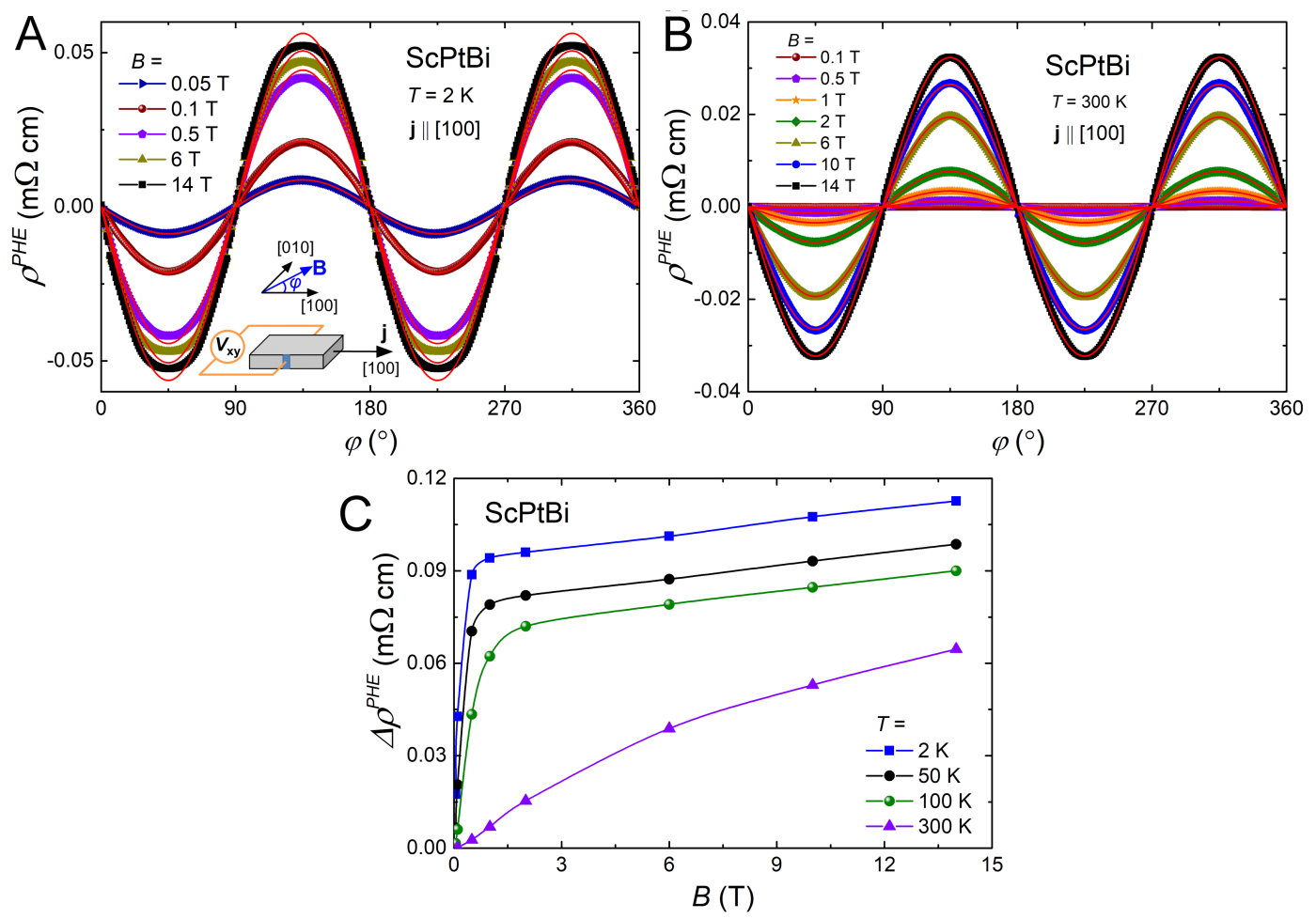

FIG. 3. Angular dependence of the planar Hall resistivity measured in different external magnetic fields at $2 \mathrm{~K}(\mathrm{~A})$, and $300 \mathrm{~K}(\mathrm{~B})$, with experimental geometry sketched in inset to panel (A). Red solid lines represent the fits obtained using Eq. (5). (C) Magnetic field variations of the PHE magnitude observed at several different temperatures. Lines are guides for the eye.

coefficient that we derived $\left(0.015 \mathrm{~T}^{-2}\right.$ at $\left.2 \mathrm{~K}\right)$ is small, but very close to $0.021 \mathrm{~T}^{-2}$ observed in isostructural YbPtBi, a well established topological semimetal 10 .

Next, we performed measurements of another indicator of CMA: the planar Hall resistivity, $\rho^{P H E}$, in the geometry shown in the inset to Fig. 3A. Magnetic field was always in plane of the sample, voltage drop was recorded as a function of angle between magnetic field and current direction, $\phi . \rho^{P H E}$ depends on angle $\phi$ as follows 5132 :

$$
\rho^{P H E}(\phi)=-\Delta \rho^{P H E} \sin 2 \phi,
$$

where $\Delta \rho^{P H E}$ stands for the magnitude of PHE. We found that behavior of $\rho^{P H E}(\phi)$ in $\mathrm{ScPtBi}$ is typical, and persists in temperatures up to 300K (Fig. 3B).

Remarkably, PHE in ScPtBi is discernible even in very weak magnetic fields: $B=0.05 \mathrm{~T}$ at $T=2 \mathrm{~K}$ (see Fig. 3A) and $B=0.1 \mathrm{~T}$ at $T=300 \mathrm{~K}$ ( Fig. $3 \mathrm{~B}$ ), indicating this material as a possible directional magnetic field sensor. $\Delta \rho^{P H E}$ measured for ScPtBi increases with increasing the strength of applied magnetic field and decreases with increasing temperature, 

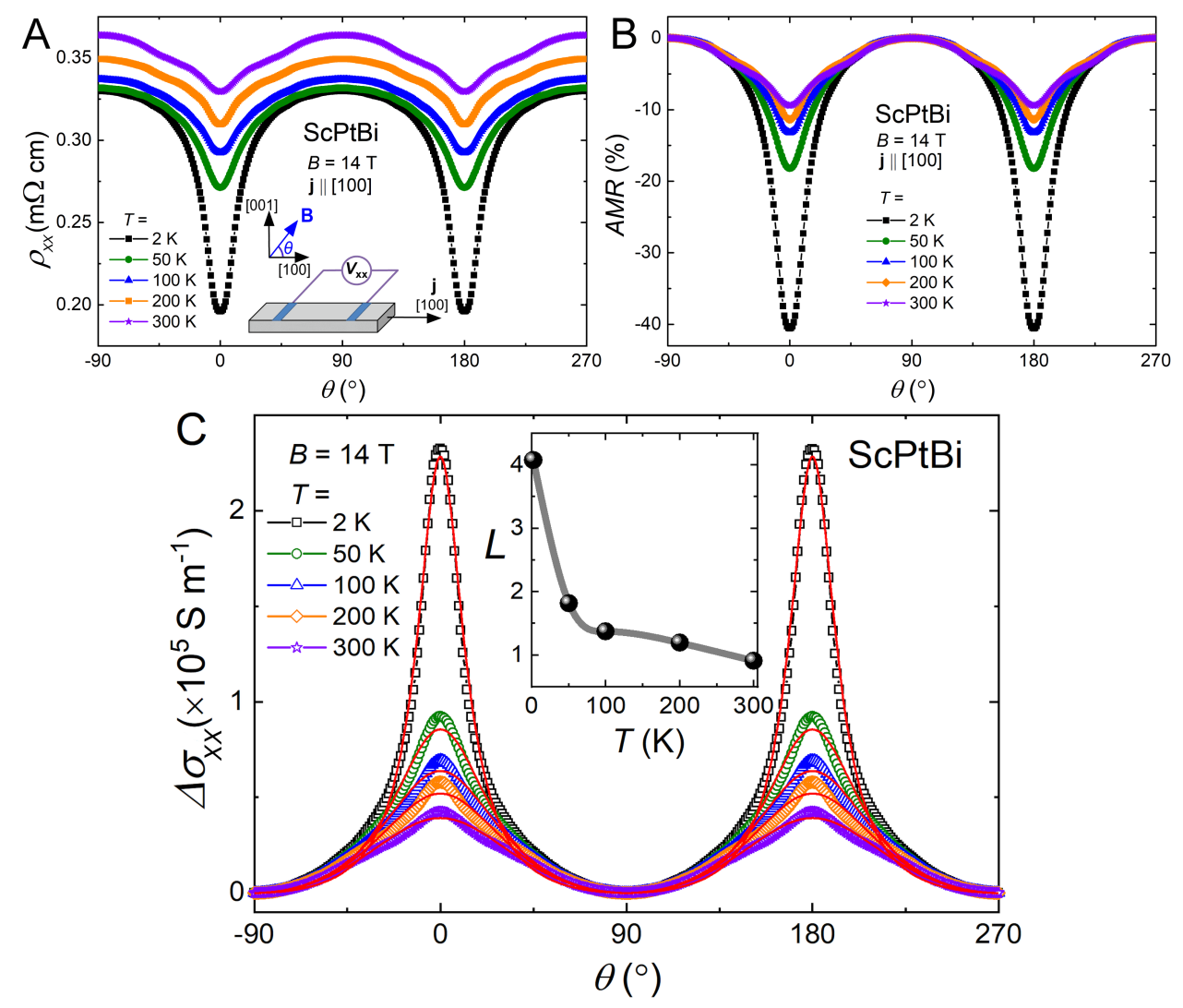

FIG. 4. (A) Angular dependence of the electrical resistivity measured at several temperatures in a magnetic field of $14 \mathrm{~T}$. The inset shows the geometry of measurement. (B) Anisotropic magnetoresistance isotherms calculated from the data presented in (A). (C) Conductivity enhancement derived from the data shown in (A). Red lines represent the results of fitting Eq. (6) to the experimental data. The inset displays the temperature dependence of the narrowing parameter $L$ defined in the text. The solid line is a guide for the eye.

which is summarized in Fig. $3 \mathrm{C}$. The magnitude of PHE in ScPtBi is smaller than reported for other half-Heusler compounds 14115 , but larger than observed for $\mathrm{VAl}_{3}$ or $\mathrm{WTe}_{2}$ 12[37.

The observation of PHE cannot be considered as sufficient proof of the existence of CMA, because any material which demonstrates anisotropic magnetoresistance (AMR) also shows PHE. AMR can have several different origins: ferromagnetism, Fermi surface anisotropy ${ }^{32}$, and/or CMA 2/3. In ferromagnets, AMR depends on $\theta$ as $\cos ^{2} \theta$, while in materials with quasi-2D Fermi surface it depends on $\theta$ as $|\cos \theta|^{\sqrt[32]{2}}$, where $\theta$ is the angle between $\mathbf{B}$ and $\mathbf{j}$.

In the presence of CMA, PHE must be accompanied by negative LMR and a particular angular dependence of AMR (also called angular narrowing of LMR) ${ }^{5}$. According to Burkov, 
the conductivity enhancement defined as $\Delta \sigma_{x x} \equiv \rho_{x x}^{-1}(\theta)-\rho_{\perp}^{-1}\left(\right.$ where $\rho_{\perp} \equiv \rho_{x x}\left(90^{\circ}\right)$ ), should depend on $\theta$ in the following way:

$$
\Delta \sigma_{x x} \propto \frac{L^{2} \cos ^{2} \theta}{1+L^{2} \sin ^{2} \theta}
$$

with $L=L_{c} / L_{a}$, where $L_{c}$ is the chiral charge diffusion length, and $L_{a}$ is the magnetic field related length scale 5 .

Thus, we measured $\rho_{x x}$ of $\mathrm{ScPtBi}$ as a function of $\theta$ in constant field $B=14 \mathrm{~T}$ at temperatures in the range from $2 \mathrm{~K}$ to $300 \mathrm{~K}$ (see Fig. $4 \mathrm{~A}$ ). Next we calculated AMR= $\left(\rho_{x x}(\theta)-\rho_{\perp}\right) / \rho_{\perp}$, which is plotted in Fig. $4 \mathrm{~B}$, and obviously follows neither $\cos ^{2} \theta$ nor $|\cos \theta|$.

However the model proposed by Burkov ${ }^{5}$ does not include WAL contribution to conductivity, which as we showed above is important in ScPtBi. Because in single-Weyl SMs $\sigma_{x x}^{W A L}$ is isotropic $\frac{35}{35}$, it will cancel out in $\Delta \sigma_{x x}(\theta)$ defined just as a difference of conductivities: $\sigma_{x x}(\theta)-\sigma_{x x}\left(90^{\circ}\right)$. Consequently, we calculated $\sigma_{x x}(\theta)=\rho_{x x}(\theta) /\left(\rho_{x x}^{2}(\theta)+\rho_{x y}^{2}(\theta)\right)$, using $\rho_{x x}(\theta)$ measured in field of $14 \mathrm{~T}$ (black symbols in Fig. 4A) and $\rho_{x y}(\theta)=\rho_{x y}(14 \mathrm{~T}) \sin \theta$, where $\rho_{x y}(14 \mathrm{~T})$ were extrapolated linearly to $B=14 \mathrm{~T}$, values of $\rho_{x y}(B)$ measured at each temperature (shown in Fig. 1A).

We fitted Eq. 6 to all $\Delta \sigma_{x x}(\theta)$ isotherms, as shown by red solid lines in Fig. 4 C. Very good fit obtained for the data collected at $T=2 \mathrm{~K}$ confirms the angular narrowing of LMR, and therefore the presence of CMA in ScPtBi.

The fit parameter $L$ "determines the strength of the chiral anomaly induced magnetotransport effects" [5. It is the ratio of two length parameters: $L_{c}=\sqrt{D \tau_{c}}$ and $L_{a}=D /(\Gamma B)$, where $D$ is diffusion coefficient of carriers (increasing with $T$ ), $\Gamma$ - parameter inversely proportional to density of states at Fermi level (nearly $T$-independent), and $\tau_{c}$ - the chiral charge relaxation time, slowly (because of topological protection and conservation of chiral charge) decreasing with temperature. Therefore, $L=\left(L_{c} / L_{a}\right) \propto 1 / \sqrt{D}$ should decrease with increasing temperature, as it is indeed shown in inset to Fig. 4C.

With increasing temperature, quality of the fitting worsens, as the contribution of CMA to LMR is reduced, which is reflected in decrease of the fitted parameter $L, \Delta \rho^{P H E}(T)$ (Fig. 3C) and $C_{W}(T)$ (inset to Fig. 2D).

Values and form of $\Delta \sigma_{x x}(\theta)$ we observed are very similar to those reported for the Weyl semimetal $\mathrm{Na}_{3} \mathrm{Bi}$, which further supports the presence of topologically non-trivial electronic structure in ScPtBi. 


\section{Electronic structure calculations}

Earlier electronic structure calculations have suggested that ScPtBi is a zero-gap semiconductor with inverted bands near the center of Brillouin zone, where the gap closes 16|17, similar to that of GdPtBi 7 .

We got very similar results for a model with perfectly ordered ScPtBi crystal structure. The obtained band structure is shown in Fig. 5A and the density of states (DOS) as a function of energy has zero value at the Fermi level, as shown in Fig. 5B. This structure is, however, in disagreement with our experimental data (metallic-like resistivity - Supplemental Fig. S4A, and specific-heat analysis in Supplemental Material ${ }^{19}$ ), which directly point to a substantial DOS at the Fermi level $\left(E_{\mathrm{F}}\right)$.

There has recently been proposed a model of half-Heusler crystal structure with Pt atom in Wyckoff position $4 c(1 / 4,1 / 4,1 / 4)$ split in randomly quarter-occupied $16 e$ position $(x, x, x)^{38}$. Refinement of X-ray diffraction pattern revealed that this model describes structure of our ScPtBi crystals equally well as the model of ideal half-Heusler (cf. Supplemental Table S1 19). We incorporated this model in our electronic structure calculations and found that relatively big change of Pt position (to $x=0.2$, i.e. 0.05 shift from $4 c$ ) results in finite $\operatorname{DOS}\left(E_{\mathrm{F}}\right)$ equal to 0.069 st./(eV f.u.) (see Figs. 5C and 5D). Our ScPtBi crystal structure refinement (Supplemental Table S1) using that model gave $x=0.242$ for $16 e$ position of Pt atoms, i.e. only 0.008 deviation from $x=1 / 4$ of $4 c$ position, much smaller than needed to sufficiently affect DOS. Therefore, this model alone does not suffice to explain semimetallic nature of $\mathrm{ScPtBi}$. Interestingly, band structure for this model contains three bands crossing Fermi level, and an gap (of $\approx 50 \mathrm{meV}$ ) between inverted bands, where Dirac points may occur (marked with a red ellipse in Fig. 5C), indispensable for realization of Weyl semimetal.

GdPtBi is a Weyl semimetal with Weyl nodes induced by external magnetic field. Magnetic field is not taken into account in ab initio band structure calculations (showing zero-gap semiconductor), and in zero-field GdPtBi does not show metallic $\rho_{x x}(T)^{7}$. Therefore, it is the influence of magnetic field on electronic structure, acting as a source of finite $\operatorname{DOS}\left(E_{\mathrm{F}}\right)$, that is a prerequisite to semimetallic state ${ }^{7}$. For $\operatorname{ScPtBi}$ metallic-like $\rho_{x x}(T)$ is observed even in zero magnetic field, which allows us to discard that scenario.

The ideal half-Heusler structure consists of three sub-lattices, each fully occupied by atoms of only one kind. But in real, even apparently perfect single crystals, different defects, 
such as antisites or vacancies, always occur. Thus, we examined if structural defects can be alternative sources of finite $\operatorname{DOS}\left(E_{\mathrm{F}}\right)$ in $\mathrm{ScPtBi}$.

Calculations for models of ScPtBi with different antisites indeed brought finite $\operatorname{DOS}\left(E_{\mathrm{F}}\right)$ (see Fig. $5 \mathrm{C}$ ) and thus profound changes in the electronic structure. For one Sc-Bi antisite per supercell, $\operatorname{DOS}\left(E_{\mathrm{F}}\right)$ is 0.83 st./(eV f.u.), for a Sc-Pt antisite: 0.60 st./(eVf.u.) and for Pt-Bi: 0.58 st./(eV f.u.), where f.u. stands for formula-unit. Vacancies also lead to non-zero values of $\operatorname{DOS}\left(E_{\mathrm{F}}\right)$. One Pt- or Bi-vacancy per supercell yielded the DOS shown in Fig. $5 \mathrm{~F}$, which at the Fermi level has values of 0.67 st./(eV f.u.), and 0.57 st./(eV f.u.), respectively (but zero for a Sc-vacancy).

All these finite $\operatorname{DOS}\left(E_{\mathrm{F}}\right)$ values are significantly larger than 0.09 st./(eVf.u.) obtained from the specific heat results (shown in Supplemental Material ${ }^{19}$ ), most likely due to smaller degree of disorder in our real crystal than that assumed in models tested. However, regardless of antisite type and for Pt- or Bi-vacancies, as well as for the structure with Pt occupying $16 e$ Wyckoff position, we always obtained semimetallic electronic structure. Additionally, in all these cases, Fermi level crosses both hole- and electron-like bands (Fig. $5 \mathrm{C}$ and Supplemental Fig. S8 $\frac{19}{\text { ) }}$, which is in agreement with the results of our Hall resistivity measurements (Fig. 1 and Table S2 19 ). Thus, both antisites and vacancies may be important sources of nonvanishing DOS at Fermi level in ScPtBi, and very likely also in other half-Heusler semimetals. Our calculations for antisite or vacancies did not reveal features of topological semimetal close to $E_{\mathrm{F}}$, but those for the structure with $\mathrm{Pt}$ at $16 e$ position revealed a gap between inverted bands where Dirac points might occur. ScPtBi may become Weyl semimetal upon application of magnetic field, splitting such Dirac point in two Weyl nodes, alike in $\mathrm{Na}_{3} \mathrm{Bi}$ or GdPtBi. Xiong et al. proposed Zeeman interaction to be responsible for such a splitting in $\mathrm{Na}_{3} \mathrm{Bi}{ }^{4}$, whereas for GdPtBi, both the Zeeman effect ${ }^{7}$ and, alternatively, the exchange field due to the magnetic moments of $\mathrm{Gd}^{39}$, have been suggested as direct causes of formation of Weyl nodes. In the case of ScPtBi, despite being isostructural with GdPtBi, the absence of atoms with strong magnetic moments leads to exclusion of the exchange field, and points to Zeeman splitting as the origin of Weyl nodes. 

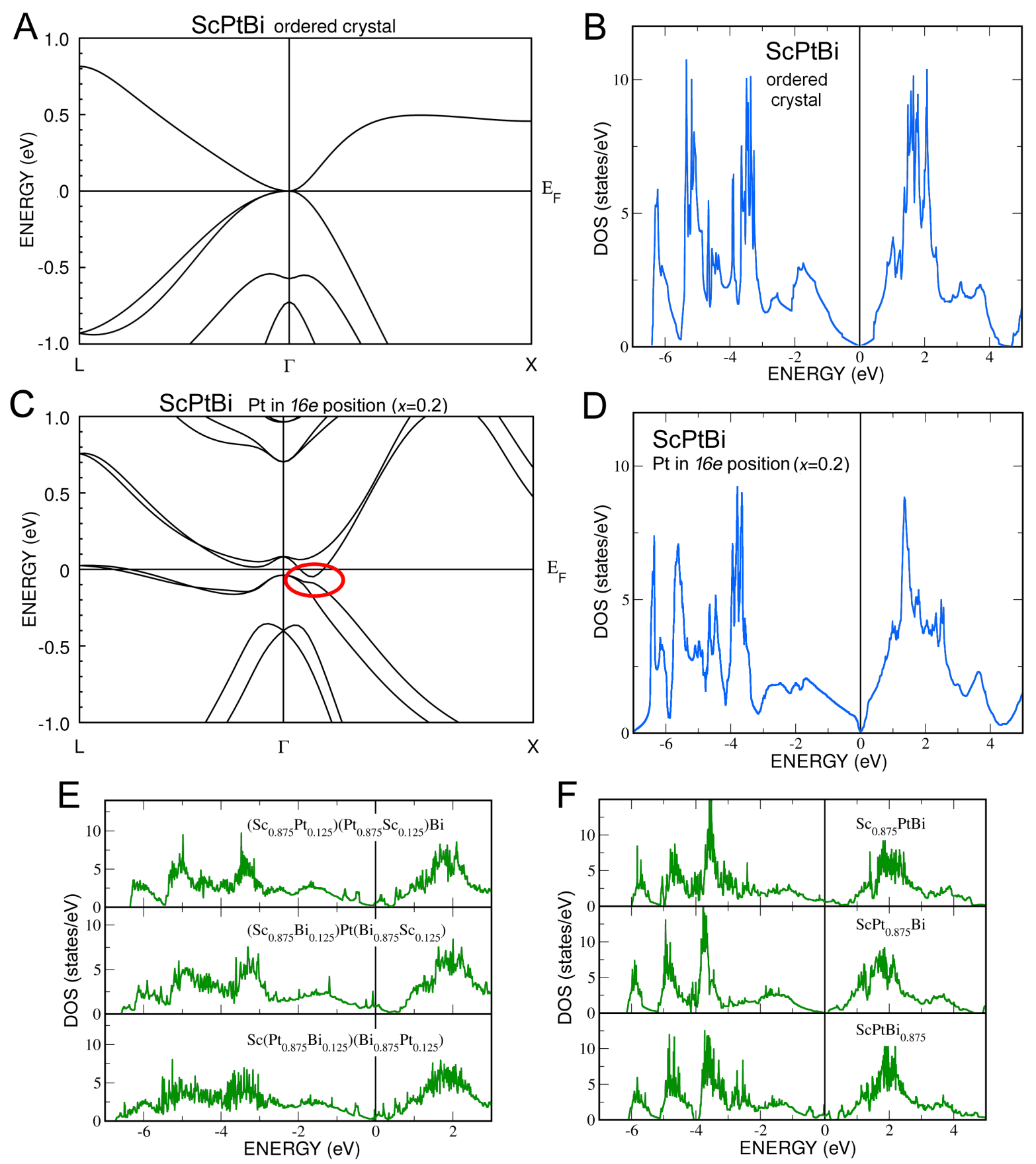

FIG. 5. (A) Band structure and (B) total DOS calculated (including spin-orbit interaction) for the ideal crystal structure of ScPtBi. (C) Band structure and (D) total DOS calculated (including spin-orbit interaction) for the model with Pt occupying the $16 e$ Wyckoff position. The red ellipse in $\mathrm{C}$ marks a gap between inverted bands where Dirac points may occur. (E,F) Total DOS for models: (E) with different antisites, and (F) with different vacancies. 


\section{CONCLUSION}

Our examination of magnetotransport in ScPtBi revealed non-linear Hall resistivity and large, positive and non-saturating TMR. Fitting the three-band Drude model to those data indicated ultrahigh carrier mobility in one of the bands. The low-field behavior of TMR could be well modeled with a WAL contribution.

Importantly, upon rotation of the sample in magnetic field, passing from a transverse to a longitudinal configuration, the slope of $\mathrm{MR}(B)$ changes from positive to negative (in fields above $3 \mathrm{~T}$ ). This reflects the significant negative component in LMR, a basic hallmark of CMA. Moreover, we observed in our sample two other crucial signs of CMA, namely the planar Hall effect and angular narrowing of negative LMR. With increasing temperature, particular parameters of all these properties: the chiral coefficient in longitudinal magnetoconductance, the magnitude of PHE and the chiral charge diffusion length clearly diminished, reflecting weakening of the CMA effect. Our analysis indicates that ScPtBi is a single-Weyl semimetal.

Our unique observation of all three hallmarks of CMA simultaneously in one material, allows us to conclude that $\mathrm{ScPtBi}$ is the new representative of topological semimetals.

The superconductivity we observed in ScPtBi makes this system even more interesting and worth further studies, due to conjunction with topologically non-trivial states, which may be important for quantum computing.

\section{Acknowledgement}

Work was supported by the National Science Centre of Poland (2015/18/A/ST3/00057 to D.K. and P.W.), and the Foundation for Polish Science (START 66.2020 to O.P.). 
* Corresponding author: p.wisniewski@intibs.pl

1 N. P. Armitage, E. J. Mele, and A. Vishwanath, Rev. Mod. Phys. 90, 015001 (2018).

2 H. Nielsen and M. Ninomiya, Phys. Lett. B 130, 389 (1983).

3 D. T. Son and B. Z. Spivak, Phys. Rev. B 88, 104412 (2013).

4 J. Xiong, S. K. Kushwaha, T. Liang, J. W. Krizan, M. Hirschberger, W. Wang, R. J. Cava, and N. P. Ong, Science 350, 413 (2015).

5 A. A. Burkov, Phys. Rev. B 96, 041110(R) (2017).

6 X. Huang, L. Zhao, Y. Long, P. Wang, D. Chen, Z. Yang, H. Liang, M. Xue, H. Weng, Z. Fang, X. Dai, and G. Chen, Phys. Rev. X 5, 031023 (2015).

7 M. Hirschberger, S. Kushwaha, Z. Wang, Q. Gibson, S. Liang, C. A. Belvin, B. A. Bernevig, R. J. Cava, and N. P. Ong, Nat. Mater. 15, 1161 (2016).

8 C.-L. Zhang, S.-Y. Xu, I. Belopolski, Z. Yuan, Z. Lin, B. Tong, G. Bian, N. Alidoust, C.-C. Lee, S.-M. Huang, T.-R. Chang, G. Chang, C.-H. Hsu, H.-T. Jeng, M. Neupane, D. S. Sanchez, H. Zheng, J. Wang, H. Lin, C. Zhang, H.-Z. Lu, S.-Q. Shen, T. Neupert, M. Zahid Hasan, and S. Jia, Nat. Commun. 7, 10735 (2016).

9 Q. Li, D. E. Kharzeev, C. Zhang, Y. Huang, I. Pletikosić, A. V. Fedorov, R. D. Zhong, J. A. Schneeloch, G. D. Gu, and T. Valla, Nat. Phys. 12, 550 (2016).

10 C. Y. Guo, F. Wu, Z. Z. Wu, M. Smidman, C. Cao, A. Bostwick, C. Jozwiak, E. Rotenberg, Y. Liu, F. Steglich, and H. Q. Yuan, Nat. Commun. 9, 4622 (2018).

11 H. Li, H.-W. Wang, H. He, J. Wang, and S.-Q. Shen, Phys. Rev. B 97, 201110(R) (2018).

12 R. Singha, S. Roy, A. Pariari, B. Satpati, and P. Mandal, Phys. Rev. B 98, 081103(R) (2018).

13 S. Liang, J. Lin, S. Kushwaha, J. Xing, N. Ni, R. J. Cava, and N. P. Ong, Phys. Rev. X 8, $031002(2018)$

14 N. Kumar, S. N. Guin, C. Felser, and C. Shekhar, Phys. Rev. B 98, 041103(R) (2018).

15 O. Pavlosiuk, D. Kaczorowski, and P. Wiśniewski, Phys. Rev. B 99, 125142 (2019).

16 S. Chadov, X. Qi, J. Kübler, G. H. Fecher, C. Felser, and S. C. Zhang, Nat. Mater. 9, 541 (2010).

17 W. Al-Sawai, H. Lin, R. S. Markiewicz, L. A. Wray, Y. Xia, S.-Y. Xu, M. Z. Hasan, and A. Bansil, Phys. Rev. B 82, 125208 (2010). 
18 Z. Hou, Y. Wang, E. Liu, H. Zhang, W. Wang, and G. Wu, Appl. Phys. Lett. 107, 202103 $(2015)$.

19 See Supplemental Material below, for results of material characterization, raw data of Hall resistance, electrical resistivity measurements, heat capacity measurement and analysis, threeband model fitting to Hall resistivity, WAL and CMA terms fitting to MR, extended electronic structure calculations, and supplemental references: $40,47$.

20 J. Rodriguez-Carvajal, Physica B 192, 55 (1993).

21 K. Koepernik and H. Eschrig, Phys. Rev. B 59, 1743 (1999).

22 H. Eschrig, K. Koepernik, and I. Chaplygin, J. Solid State Chem. 176, 482 (2003)

23 J. P. Perdew, K. Burke, and M. Ernzerhof, Phys. Rev. Lett. 77, 3865 (1996).

24 E. D. Mun, S. L. Bud'ko, C. Martin, H. Kim, M. A. Tanatar, J.-H. Park, T. Murphy, G. M. Schmiedeshoff, N. Dilley, R. Prozorov, and P. C. Canfield, Phys. Rev. B 87, 075120 (2013).

25 Z. Hou, W. Wang, G. Xu, X. Zhang, Z. Wei, S. Shen, E. K. Liu, Y. Yao, Y. Chai, Y. Sun, X. Xi, W. Wang, Z. Liu, G. Wu, and X.-X. Zhang, Phys. Rev. B 92, 235134 (2015).

26 O. Pavlosiuk, D. Kaczorowski, and P. Wiśniewski, Phys. Rev. B 94, 035130 (2016).

27 H. Xiao, T. Hu, W. Liu, Y. L. Zhu, P. G. Li, G. Mu, J. Su, K. Li, and Z. Q. Mao, Phys. Rev. B 97, 224511 (2018)

28 H. Chi, C. Zhang, G. Gu, D. E. Kharzeev, X. Dai, and Q. Li, New J. Phys. 19, 015005 (2017).

29 C. L. Zhang, Z. Yuan, Q. D. Jiang, B. Tong, C. Zhang, X. C. Xie, and S. Jia, Phys. Rev. B 95, $085202(2017)$

30 M. N. Ali, L. Schoop, J. Xiong, S. Flynn, Q. Gibson, M. Hirschberger, N. P. Ong, and R. J. Cava, Europhys. Lett. 110, 67002 (2015).

31 E. Sondheimer, Proc. R. Soc. Lond. A 268, 100 (1962).

32 A. B. Pippard, Magnetoresistance in Metals (Cambridge University Press, Cambridge, 2009).

33 J. Hu, M. M. Parish, and T. F. Rosenbaum, Phys. Rev. B 75, 214203 (2007),

34 X. Dai, H.-Z. Lu, S.-Q. Shen, and H. Yao, Phys. Rev. B 93, 161110(R) (2016).

35 H.-Z. Lu and S.-Q. Shen, Phys. Rev. B 92, 035203 (2015).

36 H. J. Kim, K. S. Kim, J. F. Wang, M. Sasaki, N. Satoh, A. Ohnishi, M. Kitaura, M. Yang, and L. Li, Phys. Rev. Lett. 111, 246603 (2013).

37 P. Li, C. Zhang, Y. Wen, L. Cheng, G. Nichols, D. G. Cory, G.-X. Miao, and X.-X. Zhang, Phys. Rev. B 100, 205128 (2019). 
K. Synoradzki, K. Ciesielski, I. Veremchuk, H. Borrmann, P. Skokowski, D. Szymański, Y. Grin, and D. Kaczorowski, Materials 12, 1723 (2019).

39 C. Shekhar, N. Kumar, V. Grinenko, S. Singh, R. Sarkar, H. Luetkens, S.-C. Wu, Y. Zhang, A. C. Komarek, E. Kampert, Y. Skourski, J. Wosnitza, W. Schnelle, A. McCollam, U. Zeitler, J. Kübler, B. Yan, H.-H. Klauss, S. S. P. Parkin, and C. Felser, Proc. Natl. Acad. Sci. USA 115, 9140 (2018).

40 Y. Nakajima, R. Hu, K. Kirshenbaum, A. Hughes, P. Syers, X. Wang, K. Wang, R. Wang, S. R. Saha, D. Pratt, J. W. Lynn, and J. Paglione, Sci. Adv. 1, e1500242 (2015).

41 O. Pavlosiuk, D. Kaczorowski, and P. Wiśniewski, Sci. Rep. 5, 9158 (2015).

42 O. Pavlosiuk, D. Kaczorowski, X. Fabrèges, A. Gukasov, and P. Wiśniewski, Sci. Rep. 6, 18797 $(2016)$.

43 N. P. Butch, P. Syers, K. Kirshenbaum, A. P. Hope, and J. Paglione, Phys. Rev. B 84, 220504(R) (2011).

44 Y. Wu, N. H. Jo, M. Ochi, L. Huang, D. Mou, S. L. Bud'ko, P. C. Canfield, N. Trivedi, R. Arita, and A. Kaminski, Phys. Rev. Lett. 115, 166602 (2015).

45 Y. Zhang, C. Wang, L. Yu, G. Liu, A. Liang, J. Huang, S. Nie, X. Sun, Y. Zhang, B. Shen, J. Liu, H. Weng, L. Zhao, G. Chen, X. Jia, C. Hu, Y. Ding, W. Zhao, Q. Gao, C. Li, S. He, L. Zhao, F. Zhang, S. Zhang, F. Yang, Z. Wang, Q. Peng, X. Dai, Z. Fang, Z. Xu, C. Chen, and X. J. Zhou, Nat. Commun. 8, 15512 (2017).

46 G. Ding, G. Y. Gao, L. Yu, Y. Ni, and K. Yao, J. Appl. Phys. 119, 025105 (2016).

47 K. Kaur, S. Dhiman, and R. Kumar, Phys. Lett. A 381, 339 (2017). 
Supplemental Material for

\title{
Magnetotransport signatures of chiral magnetic anomaly in the half-Heusler phase ScPtBi
}

\author{
Orest Pavlosiuk ${ }^{1}$, Andrzej Jezierski ${ }^{2}$, Dariusz Kaczorowski ${ }^{1}$ and Piotr Wiśniewski ${ }^{1}$ \\ ${ }^{1}$ Institute of Low Temperature and Structure Research, Polish Academy of Sciences, \\ 50-422 Wrockaw, Poland \\ ${ }^{2}$ Institute of Molecular Physics, Polish Academy of Sciences, 60-179 Poznań, Poland
}

\section{Contents}

Figure S1 - Energy-dispersive X-ray spectrum.

Powder and Laue X-ray diffraction results (incl. Figure S2 and Table S1).

Specific heat capacity results (incl. Figure S3).

Electrical resistivity and transverse magnetoresistance results (incl. Figure S4).

Figure S5 - Raw data of Hall resistance.

Three-band model analysis of Hall conductivity (incl. Figure S6 and Table S2).

Weak antilocalization analysis and CMA parameters for different temperatures (incl. Figure S7 and Table S3)

Supplementary results of electronic structure calculations (incl. Figs. S8 and S9).

Supplementary references. 


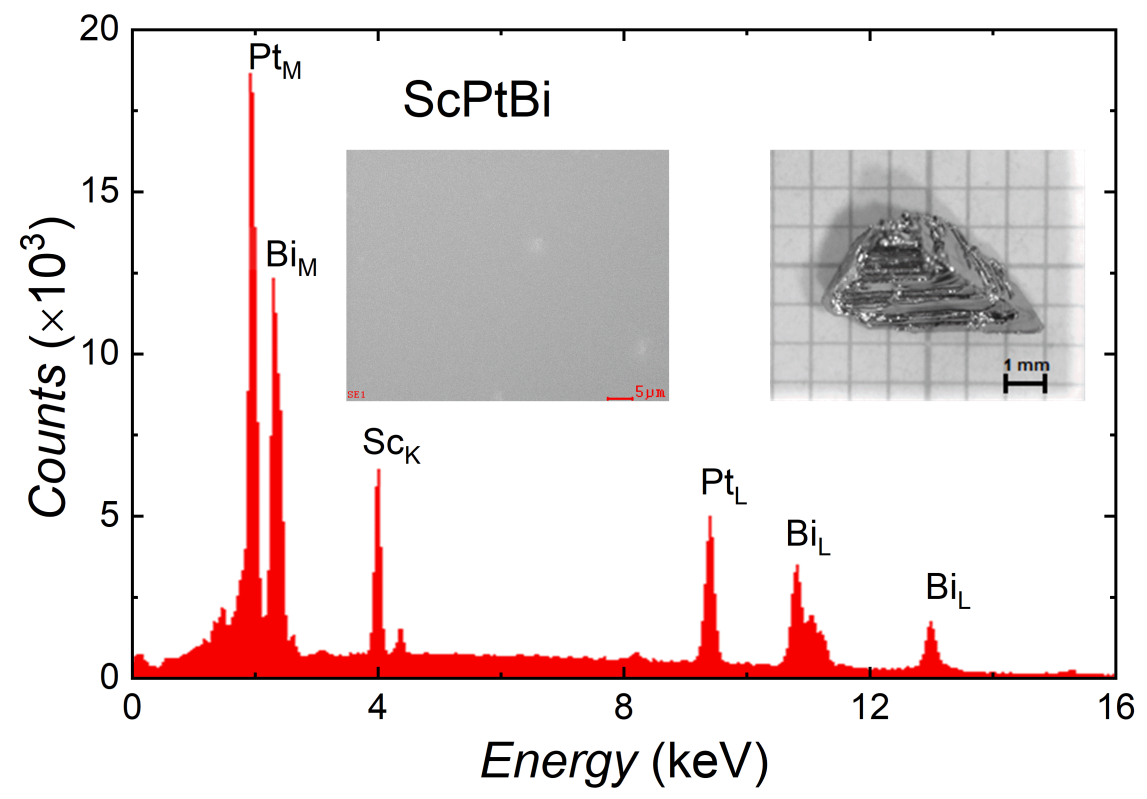

FIG. S1. Energy-dispersive X-ray spectrum (EDS), SEM image (left inset) and photograph (right inset) of ScPtBi single crystal. Chemical composition determined from the spectrum is close to the equiatomic: $\mathrm{Sc}_{33.33} \mathrm{Pt}_{34.03} \mathrm{Bi}_{32.64}$.

\section{Powder and Laue X-ray diffraction results}

Figure S2A demonstrates an X-ray diffractogram of powdered ScPtBi (collected at $300 \mathrm{~K}$ ) and results of Rietveld refinement of its crystal structure. All obtained Bragg peaks could be indexed with the $F \overline{4} 3 m$ space group, typical for half-Heusler phases. Crystal structure data obtained from the refinement are gathered in Table S1, where model 1 represents ideal half-Heusler crystal structure.

Several models of crystal structure have been taken into consideration in the refinement. Following the idea from [1], we replaced fully occupied position of Pt atom $4 c(1 / 4,1 / 4,1 / 4)$ with quarter-filled position $16 e(x, x, x)$, this is denoted as model 2 in Table S1. Our attempts to use other models which consider different type of crystal structure disorder (vacancies and antisites) did not yield statistically significant results, most likely because of accuracy limitations of our standard X-ray diffractometer.

Laue backscattering technique confirmed high quality of studied single crystal and allowed precise orientation and cutting of prism-shaped samples for electronic-transport measurements. Figure S2B shows an exemplary Laue pattern. 

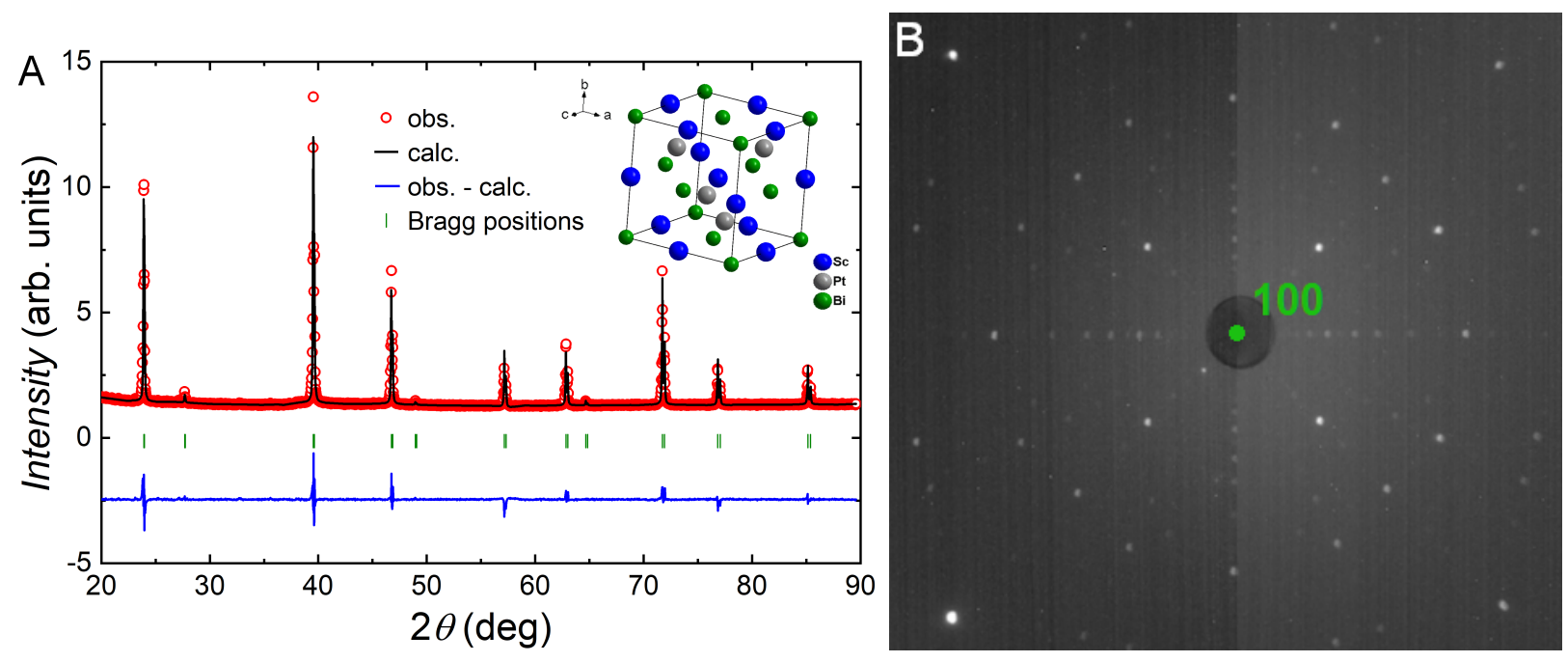

FIG. S2. (A) X-ray diffractogram of powdered single crystals of ScPtBi. Inset shows the unit cell. (B) Laue diffraction pattern for ScPtBi single crystal taken with incident X-ray beam along the [100] crystallographic direction.

TABLE S1 Crystal data and structure refinement results for ScPtBi.

\begin{tabular}{|c|c|c|}
\hline & model 1 & model 2 \\
\hline space group & \multicolumn{2}{|c|}{$F \overline{4} 3 m$} \\
\hline lattice parameter, A & $6.441268(36)$ & $6.441257(37)$ \\
\hline formula units per unit cell & \multicolumn{2}{|c|}{4} \\
\hline Sc Wyckoff position & \multicolumn{2}{|c|}{$4 b(1,1,1)$ (fully occupied) } \\
\hline \multirow[t]{3}{*}{ Pt Wyckoff position } & \multirow{3}{*}{$\begin{array}{l}4 c(1 / 4,1 / 4,1 / 4) \\
\text { (fully occupied) }\end{array}$} & $16 e(x, x, x)$ \\
\hline & & (occupied in $25 \%$ ) \\
\hline & & with $x=0.2421(25)$ \\
\hline Bi Wyckoff position & \multicolumn{2}{|c|}{$4 a(0,0,0)$ (fully occupied) } \\
\hline$B_{\text {iso }} \mathrm{Sc}$ & $1.65(38)$ & $1.53(38)$ \\
\hline$B_{\text {iso }} \mathrm{Pt}$ & $0.82(20)$ & $0.63(20)$ \\
\hline$B_{\text {iso }} \mathrm{Bi}$ & $0.09(17)$ & $0.08(17)$ \\
\hline Bragg $R$-factor, $\%$ & 5.33 & 5.22 \\
\hline$R_{f}$-factor, $\%$ & 3.21 & 3.24 \\
\hline
\end{tabular}




\section{Specific heat capacity}

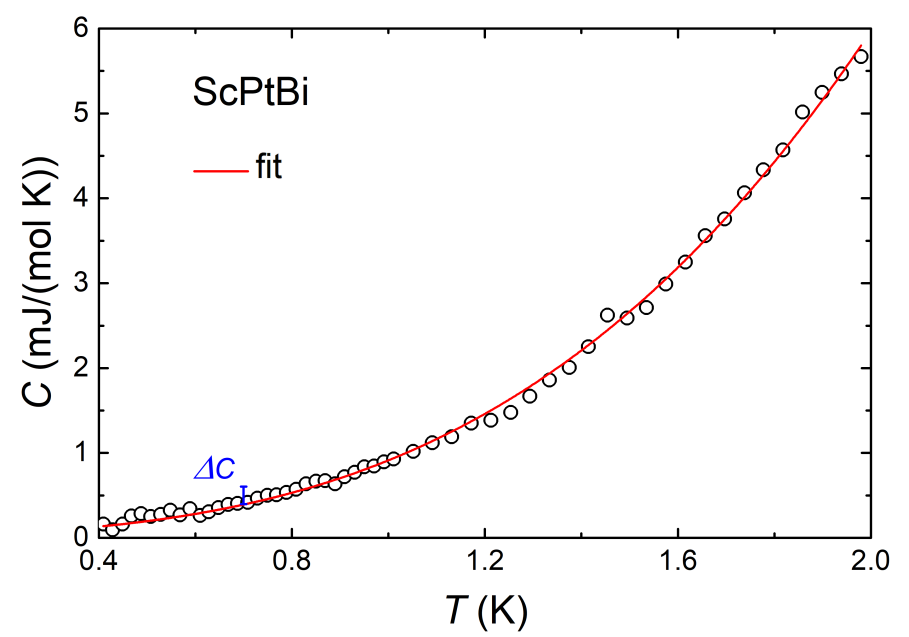

FIG. S3. Temperature dependent specific heat of ScPtBi. Red solid line corresponds to the fit with $C=\gamma T+\beta T^{3}$ equation. Blue bar corresponds to the specific heat jump expected for a conventional superconductor.

Temperature dependence of specific heat (Fig. S3) shows no clear anomaly, which could be attributed to the superconducting phase transition. This is common feature for all halfHeusler superconductors which contain rare earth elements and bismuth [2-6]. $C(T)$ is well described by a standard expression $C=\gamma T+\beta T^{3}$, where $\gamma T$ corresponds to electronic contribution and $\beta T^{3}$ to phonon contribution to the total specific heat. The obtained fitting parameters are: $\gamma=0.22 \mathrm{~mJ} /\left(\mathrm{mol} \mathrm{K}^{2}\right)$ and $\beta=0.69 \mathrm{~mJ} /\left(\mathrm{mol} \mathrm{K}^{4}\right)$. From the value of $\beta$ we obtained Debye temperature: $\Theta_{\mathrm{D}}=\left(36 R \pi^{4} / 5 \beta\right)^{1 / 3}=204 \mathrm{~K}$, which is similar to those reported for other half-Heusler phases $[3,4]$. Sommerfeld coefficient, $\gamma$, is proportional to density of states (DOS) at Fermi level: $\operatorname{DOS}\left(E_{\mathrm{F}}\right)=3 \gamma\left(\pi k_{\mathrm{B}}\right)^{-2}$, where $k_{\mathrm{B}}$ is Boltzmann constant. Using this formula we obtained $\operatorname{DOS}\left(E_{\mathrm{F}}\right)=0.09 \mathrm{st} . /(\mathrm{eV} \mathrm{f.u})$, which is equivalent to $1.41 \times 10^{47} \mathrm{st} . /\left(\mathrm{eV} \mathrm{m}^{-3}\right)$. In turn, carrier concentration, $n$, is proportional to $\operatorname{DOS}\left(E_{\mathrm{F}}\right)$ $\left(n=2 / 3 \operatorname{DOS}\left(E_{\mathrm{F}}\right)\right.$. Assuming that Fermi energy of ScPtBi is similar to that of YPtBi (estimated between 13 and $71 \mathrm{meV}[6,7]$ ), we got $n \approx 10^{19} \mathrm{~cm}^{-3}$. Its order of magnitude is in very good agreement with carrier concentration values obtained from the three-bands model analysis of the Hall conductivity (Table S2). Considering that mechanism of superconductivity in ScPtBi can be described by BCS theory, we calculated the value of jump in $C\left(T_{\mathrm{c}}\right)$, which equals to $\Delta C=1.43 \gamma T_{\mathrm{c}}=0.21 \mathrm{~mJ} /(\mathrm{mol} \mathrm{K})$. This jump, shown as a blue error bar in Fig. S3, is very small, and thus could not be resolved in our experiment. 


\section{Electrical resistivity and transverse magnetoresistance}
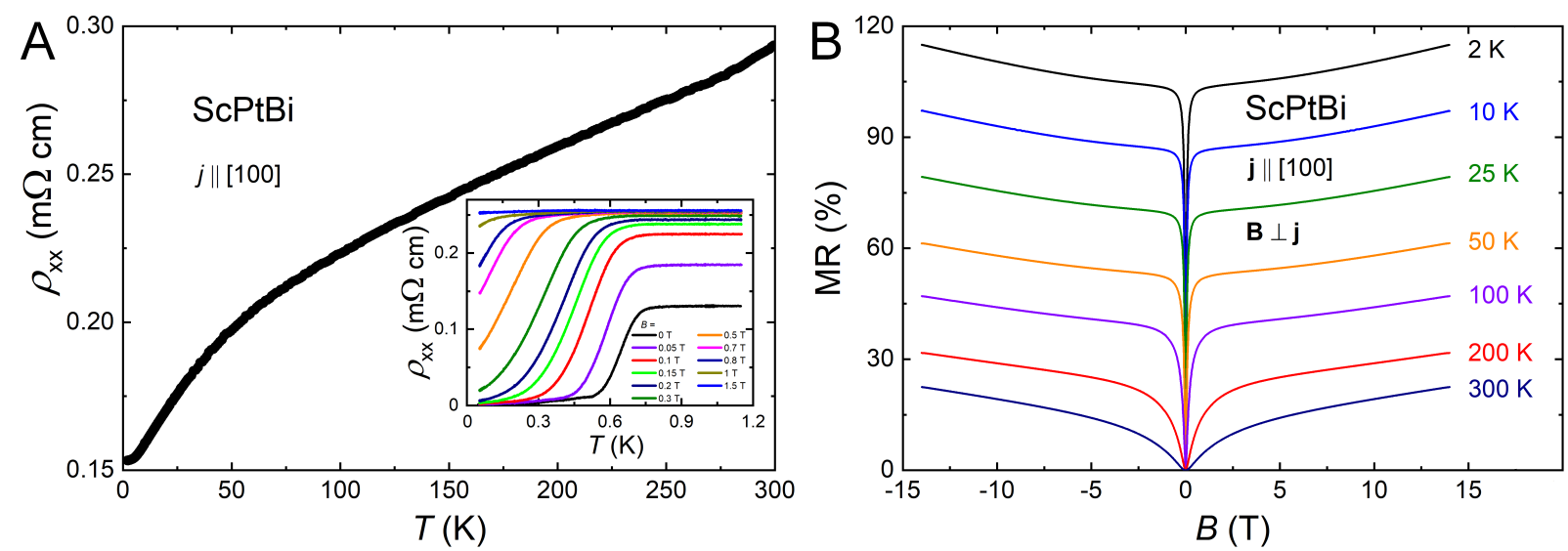

FIG. S4. (A) Temperature dependence of the electrical resistivity measured with electric current j $\|$ [100]. Inset shows electrical resistivity in low temperature range measured in magnetic fields of different strength aapplied transverse to the current. (B) Transverse MR measured at several different temperatures as a function of magnetic field.

Electrical resistivity, $\rho_{x x}$, of ScPtBi exhibits metallic-like temperature dependence (Fig. S4A). Shape of the $\rho_{x x}(T)$ curve and the ratio $\rho_{x x}(300 \mathrm{~K}) / \rho_{x x}(2 \mathrm{~K}) \approx 2$ are similar to those reported before [8], however distinctly different from $\rho_{x x}(T)$ of GdPtBi, which electronic structure [9] was supposed to closely resemble that of ScPtBi. Moreover, below 0.7 K, $\rho_{x x}$ decreases sharply by about $90 \%$, then, below $0.5 \mathrm{~K}$, it undergoes another less abrupt decrease, down to zero value at $T=0.23 \mathrm{~K}$. Similar two-step superconducting transitions have previously been reported for numerous half-Heusler superconductors [2]. The critical temperature, $T_{\mathrm{c}}$, decreases with increasing strength of applied magnetic field, and at $0.05 \mathrm{~K}$ the superconductivity becomes totally suppressed by a magnetic field of $1.5 \mathrm{~T}$ (inset to Fig. S4A). We thoroughly surveyed the literature and found that no binary compound of Sc, Pt and $\mathrm{Bi}$ is superconducting with $T_{\mathrm{c}}$ similar to that observed for our ScPtBi sample. Moreover, scanning electron microscopy with EDS analysis did not reveal any non-homogeneities in our crystals. Therefore, we can safely exclude the superconductivity originating from an impurity phase.

Slightly different residual resistivities $(0.16 \mathrm{~m} \Omega \mathrm{cm}$ in panel $(\mathrm{A})$ and $0.13 \mathrm{~m} \Omega \mathrm{cm}$ in the inset) occur because these data were obtained in two resistance measurements. The first was done with PPMS in its basic configuration (providing $T \geq 1.8 \mathrm{~K}$ ), in the second one we 
used PPMS with dilution refrigerator, and sample with fresh electrical contacts mounted on another sample holder. Distances between voltage contacts were slightly different in both cases, and the limited accuracy of their measurement caused this resistivity discrepancy.

Transverse magnetoresistance of ScPtBi (TMR, j \|[100], B \|[001]), as a function of magnetic field at several temperatures from $2 \mathrm{~K}$ to $300 \mathrm{~K}$, is shown in Fig. S4B. It is positive in the entire covered temperature range and does not saturate in strong magnetic fields. It increases very abruptly in weak magnetic fields: for example, at $T=2 \mathrm{~K}$ it reaches $100 \%$ already in $B=0.35 \mathrm{~T}$. Values of $\rho_{x x}$ underlying these TMR curves were used for WAL analyses of magnetoconductivity for $T=2 \mathrm{~K}$ (viz. Fig. $2 \mathrm{C}$ ), and for higher temperatures (Fig. S6 and Table S3A).

\section{Raw data of Hall resistance}

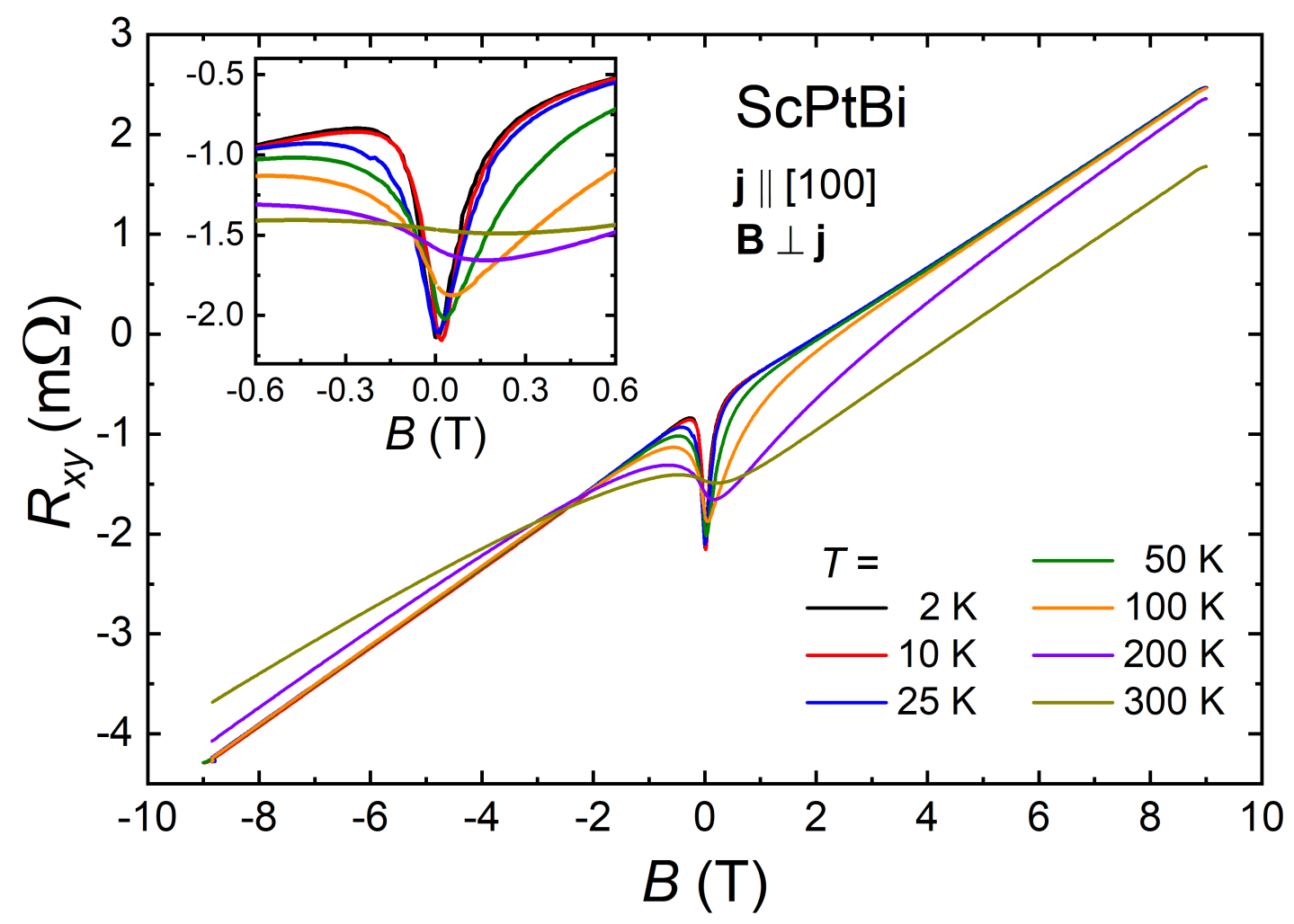

FIG. S5. Hall resistance measured at several temperatures with electric current $\mathbf{j} \|[100]$. Inset shows zoom in weak magnetic field range. Hall resistivity data shown in Fig. 1A of the main text were obtained through anti-symmetrization of data shown here, as:

$\rho_{x y}(B)=t\left(R_{x y}(B)-R_{x y}(-B)\right) / 2$, where $t$ was the thickness of the sample. 


\section{Three-band model analysis of Hall conductivity}

For a quantitative analysis of our data we used three-band Drude model of Hall conductivity (Eq. 1 of the main text):

$$
\sigma_{x y}(B)=\sum_{i=1}^{3} \frac{e n_{i} \mu_{i}^{2} B}{1+\left(\mu_{i} B\right)^{2}},
$$

with $n_{i}$ and $\mu_{i}$ denoting respectively concentration and mobility of carriers from the $i$-th band ( $e$ is the elementary charge). We fitted Eq. S1 to the experimental Hall conductivity data: $\sigma_{x y}=\rho_{x y} /\left(\rho_{x x}^{2}+\rho_{x y}^{2}\right)$ (because $\left.\rho_{x y} \ll \rho_{x x}\right)$. The results of this fitting to $\sigma_{x y}(B)$ obtained at temperatures higher than $2 \mathrm{~K}$ are shown in Fig. S6 (as red solid lines). Parameters of all fits and collected in Table S2. Interestingly, our analysis demonstrates that between $T=50 \mathrm{~K}$ and $T=100 \mathrm{~K}$ hole-like character of one of bands changes to electron-like. This behavior can possibly be due to a temperature-driven Lifshitz transition, previously observed in topological semimetals, for example $\mathrm{WTe}_{2}[10]$ and $\mathrm{ZrTe}_{5}[11,12]$.
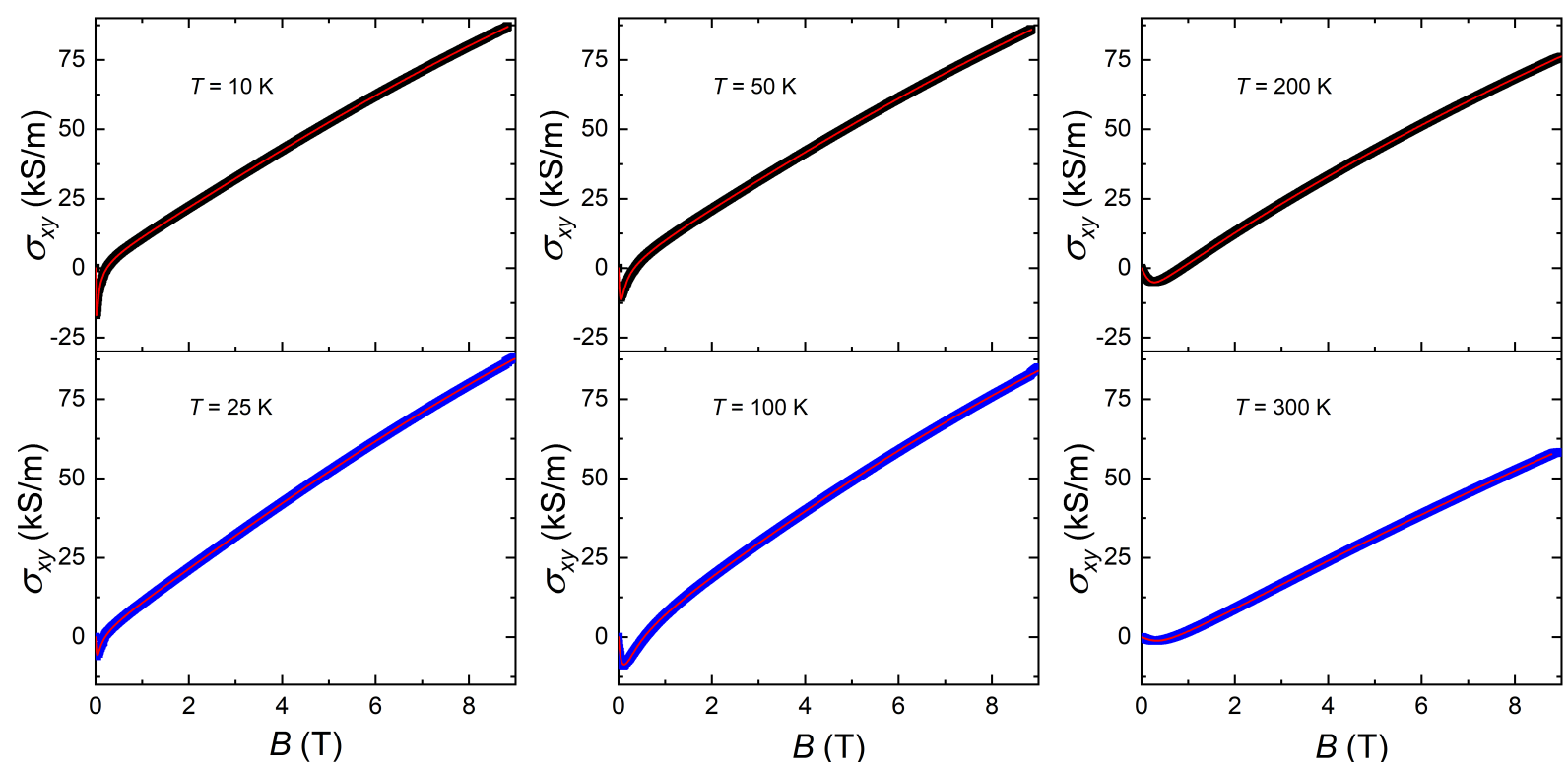

FIG. S6. Hall conductivity as a function of magnetic field for several temperatures. Red solid lines are the fits of the three-bands Drude model (Eq. S1). Parameters of the fitted models are collected in Table S2. Quality of all fits was excellent (with $R^{2}>99.99$ ). 
TABLE S2 Parameters obtained for ScPtBi from three-bands Drude model fitting to Hall conductivity shown in Fig. 1 (main text) and Fig. S6. $n_{i}$ are carrier concentrations, $\mu_{i}$ are carrier mobilities (for $i=1,2,3$ ). Carriers with positive $n_{i}$ are holes, those with negative $n_{i}$ are electrons. All fit parameters were obtained with errors smaller than $7 \%$.

\begin{tabular}{ccccccc}
\hline \hline$T$ & $n_{1}$ & $\mu_{1}$ & $n_{2}$ & $\mu_{2}$ & $n_{3}$ \\
$(\mathrm{~K})$ & $\left(\mathrm{cm}^{-3}\right)$ & $\left(\mathrm{cm}^{2} \mathrm{~V}^{-1} \mathrm{~s}^{-1}\right)$ & $\left(\mathrm{cm}^{-3}\right)$ & $\left(\mathrm{cm}^{2} \mathrm{~V}^{-1} \mathrm{~s}^{-1}\right)$ & $\left(\mathrm{cm}^{-3}\right)$ & $\mu_{3}$ \\
\hline 2 & $5.47 \times 10^{19}$ & 351 & $-4.55 \times 10^{15}$ & 212816 & $1.14 \times 10^{16}$ & 10386 \\
10 & $5.33 \times 10^{19}$ & 356 & $-4.98 \times 10^{15}$ & 435109 & $1.11 \times 10^{16}$ & 12710 \\
25 & $5.51 \times 10^{19}$ & 348 & $-3.34 \times 10^{15}$ & 229137 & $6.91 \times 10^{15}$ & 11706 \\
50 & $5.59 \times 10^{19}$ & 343 & $-8.34 \times 10^{15}$ & 179416 & $1.50 \times 10^{16}$ & 6105 \\
100 & $5.04 \times 10^{19}$ & 358 & $-2.87 \times 10^{15}$ & 166572 & $-1.90 \times 10^{16}$ & 50797 \\
200 & $6.87 \times 10^{19}$ & 290 & $-2.49 \times 10^{16}$ & 30429 & $-6.87 \times 10^{16}$ & 7577 \\
300 & $1.18 \times 10^{20}$ & 192 & $-2.25 \times 10^{16}$ & 16250 & $-9.14 \times 10^{16}$ & 4591 \\
\hline \hline
\end{tabular}


Weak antilocalization analysis and CMA parameters for different temperatures
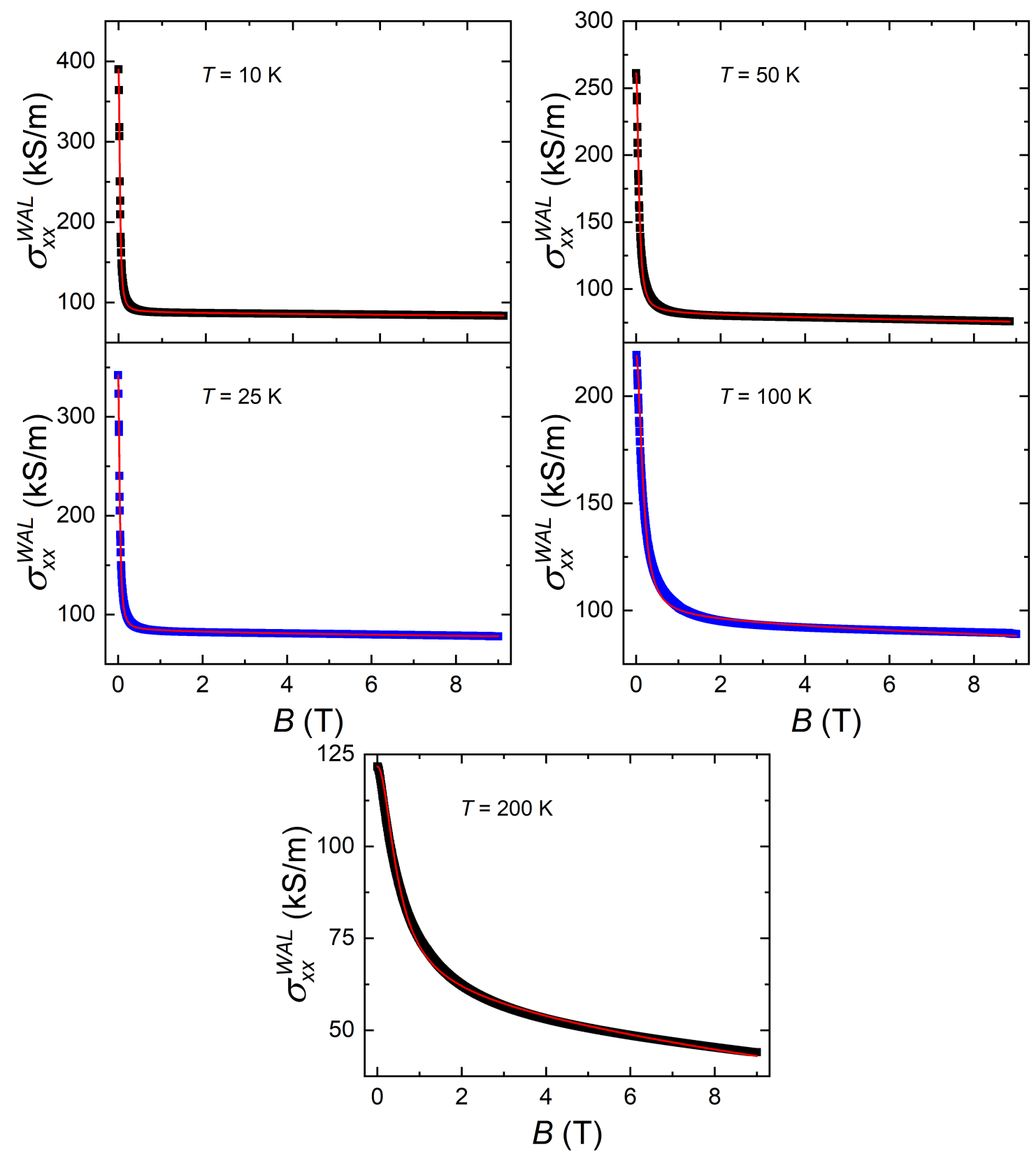

FIG. S7. Extracted contribution of weak antilocalization to longitudinal electrical conductivity of $\mathrm{ScPtBi}$ as a function of magnetic field, for several temperatures. Red lines correspond to the fits with Eq. 3 of the main text. Parameters of the fitted models are collected in Table S3A. 
TABLE S3 Weak antilocalization and chiral magnetic anomaly parameters for longitudinal electrical conductivity of ScPtBi at different temperatures

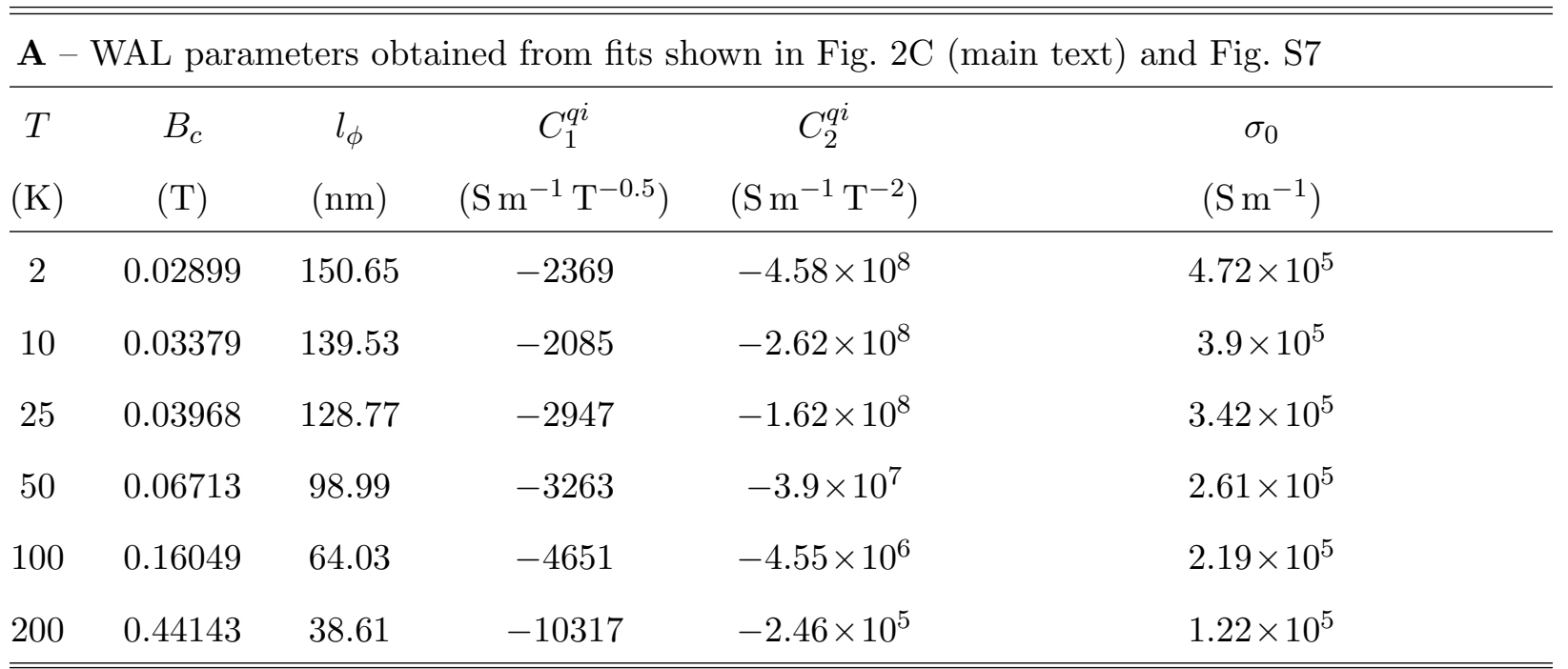

B - WAL and CMA parameters obtained from fits of Eq. 3, as shown in Fig. 2D (main text)

\begin{tabular}{|c|c|c|c|c|c|c|c|}
\hline $\begin{array}{l}T \\
(\mathrm{~K})\end{array}$ & $\begin{array}{l}B_{c} \\
(\mathrm{~T})\end{array}$ & $\begin{array}{c}l_{\phi} \\
(\mathrm{nm})\end{array}$ & $\begin{array}{c}C_{1}^{q i} \\
\left(\mathrm{~S} \mathrm{~m}^{-1} \mathrm{~T}^{-0.5}\right)\end{array}$ & $\begin{array}{c}C_{2}^{q i} \\
\left(\mathrm{~S} \mathrm{~m}^{-1} \mathrm{~T}^{-2}\right)\end{array}$ & $\begin{array}{c}\sigma_{0} \\
\left(\mathrm{~S} \mathrm{~m}^{-1}\right)\end{array}$ & $\begin{array}{c}\sigma_{n} \\
\left(\mathrm{~S} \mathrm{~m}^{-1}\right)\end{array}$ & $\begin{array}{c}C_{W} \\
\left(\mathrm{~T}^{-2}\right)\end{array}$ \\
\hline 2 & 0.08621 & 87.36 & -45434 & $-3.3 \times 10^{7}$ & $4.72 \times 10^{5}$ & $3.25 \times 10^{5}$ & 0.0137 \\
\hline 25 & 0.07837 & 91.62 & -27675 & $-3.20 \times 10^{7}$ & $3.42 \times 10^{5}$ & $3.21 \times 10^{5}$ & 0.00906 \\
\hline 50 & 0.11497 & 75.65 & -15474 & $-1.12 \times 10^{7}$ & $2.61 \times 10^{5}$ & $3.33 \times 10^{5}$ & 0.00275 \\
\hline 100 & 0.20778 & 56.27 & -14966 & $-2.1 \times 10^{6}$ & $2.19 \times 10^{5}$ & $3.12 \times 10^{5}$ & 0.00137 \\
\hline
\end{tabular}

WAL parameters obtained from analysis of TMR (A) and LMR (B) are somewhat different, however similar differences were reported for a Dirac semimetal $\mathrm{Bi}_{0.97} \mathrm{Sb}_{0.03}$ by Kim et al. [13] .

Because the conductivity related to CMA (Eq. 17 in [14]) is: $\frac{e^{4}}{4 \pi^{2} \hbar c^{2}} \frac{v^{3} \tau}{\mu_{c}^{2}} B^{2}$ we may consider $C_{W}$ proportional to $\frac{v^{3} \tau}{\mu_{c}^{2}}$ (where $v$ is Fermi velocity, $\tau$ intervalley scattering relaxation time, and $\mu_{c}$ is chemical potential measured from Weyl point). Small value of $C_{W}$ may thus implicate small $v$ and/or $\tau$, and/or large $\mu_{c}$. Large mobilities obtained from Hall effect analysis indicate large $v$ and $\tau$, therefore the implication of a small $C_{W}$ in $\mathrm{ScPtBi}$ is most likely the large value of $\mu_{c}$. 


\section{Supplementary results of electronic structure calculations}

We show in Fig. S8 band structures for models with different antisite defects, and in Fig. S9 total energy, band structure and DOS calculated for the model of tetragonally distorted ScPtBi.
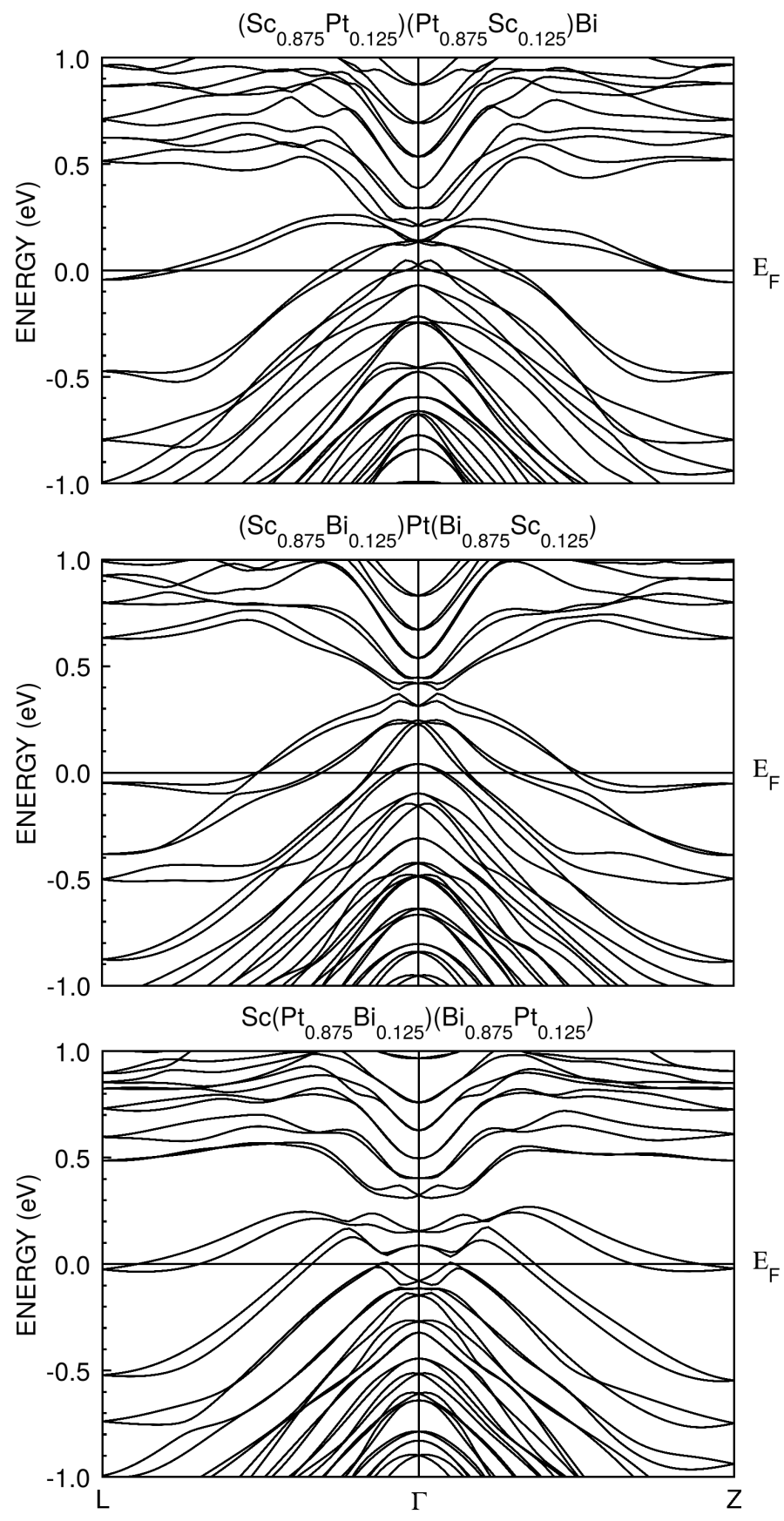

FIG. S8. Electronic band structure of ScPtBi for crystal structure models with different antisite defects. Note both hole-like and electron-like bands crossing the Fermi energy $E_{\mathrm{F}}$. 
For the model of tetragonally distorted ScPtBi we obtained results shown in Fig. S9. First, we calculated total energy for crystal structure of ScPtBi as a function of ratio of lattice parameters, $c / a$ (Fig. S9A). The lowest energy was found for $c / a=1.0287$, and for such a distortion we calculated band structure and DOS, as shown in Fig. S9B-D, revealing a gap of $23 \mathrm{meV}$. It closely resembles results presented by Ding, Gao, Yu, Ni, and Yao [15], as well as by Kaur, Dhiman, and Kumar [16]. In these both papers the increase of the $c / a$ ratio by $5 \%$ induced opening of the gap of $\approx 70 \mathrm{meV}$, however in the latter paper, for a $3 \%$ distortion the gap remained closed, most likely due to different calculation codes applied.
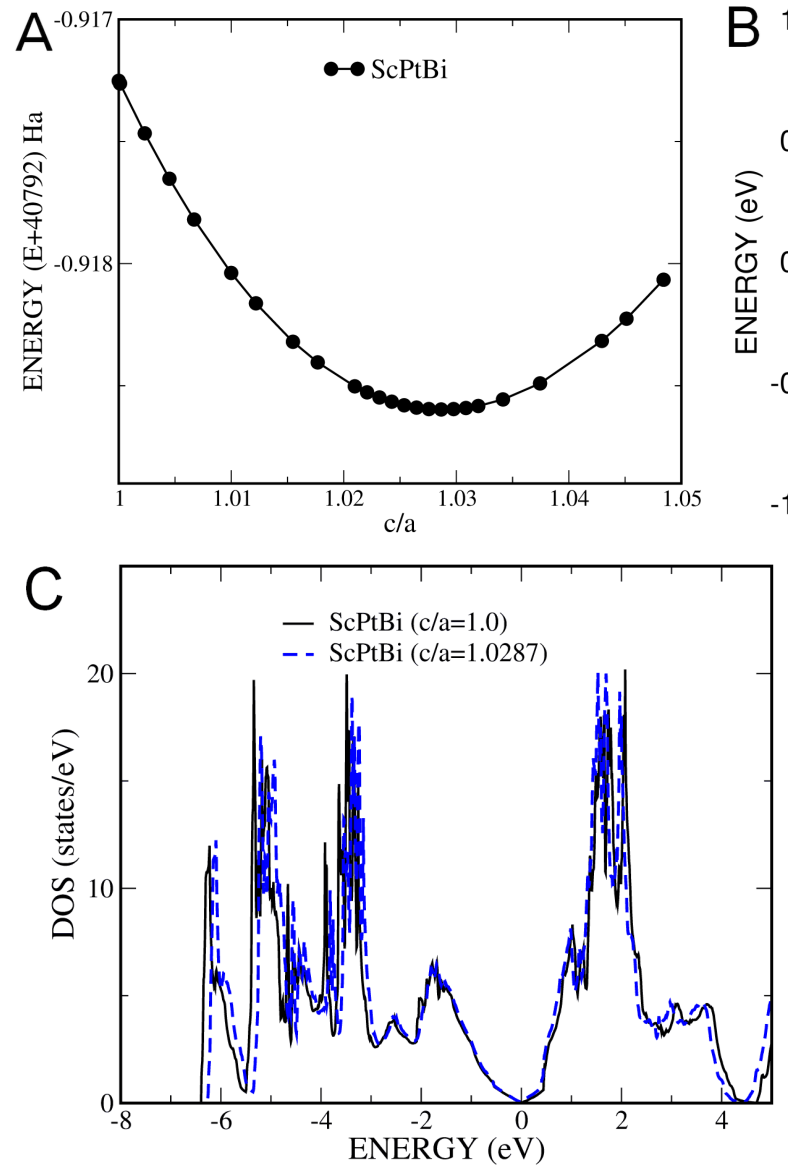
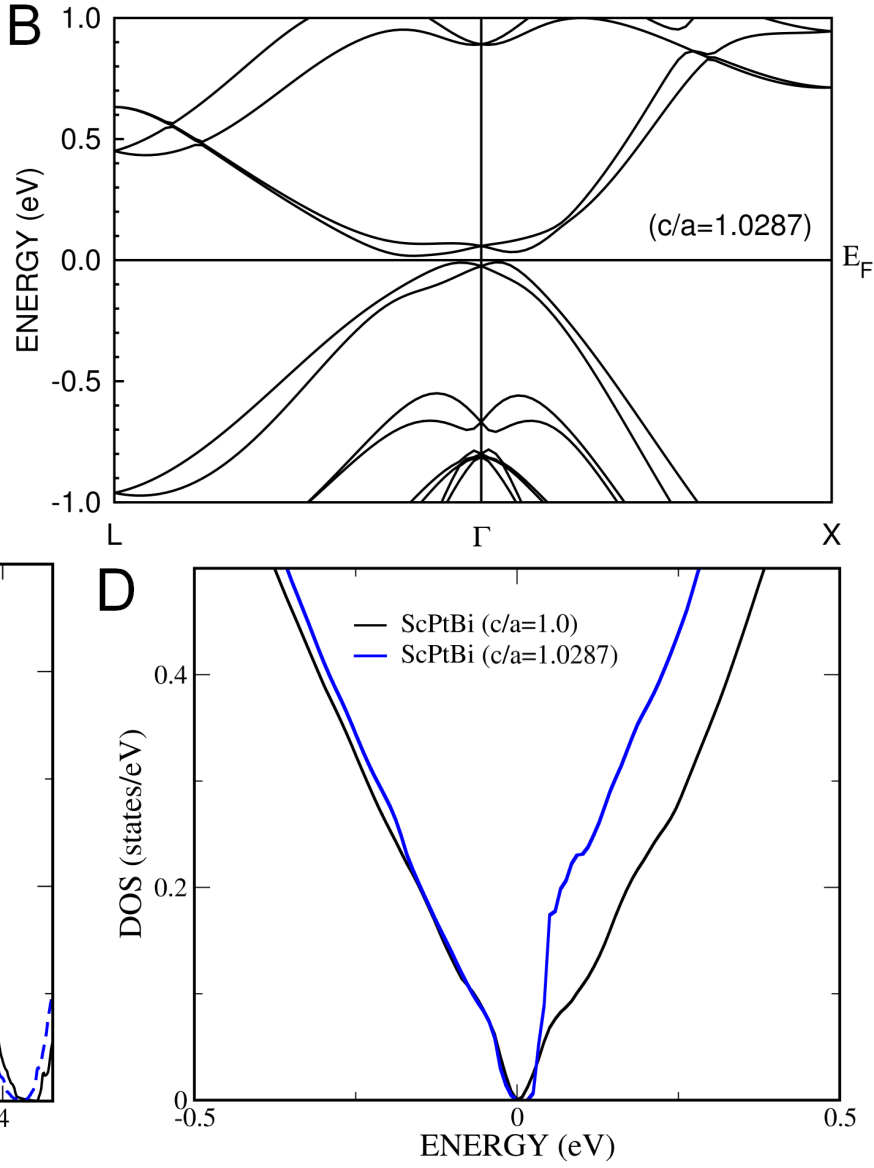

FIG. S9. (A) Total energy calculated for tetragonally distorted crystal structure of ScPtBi as a function of ratio of lattice parameters, $c / a$. (B) Electronic structure of tetragonally distorted $\mathrm{ScPtBi}$, with $c / a=1.0287$ - corresponding to energy minimum in panel (A). (C) Calculated density of states in tetragonally distorted $\mathrm{ScPtBi}$ (with $c / a=1.0287$ ) - blue dashed line, compared with density of states of ScPtBi with cubic crystal structure - black solid line. (D) Same as in (C) but in close vicinity of Fermi level. For $c / a=1.0287 \operatorname{DOS}\left(E_{\mathrm{F}}\right)$ is zero, and there is a gap of $23 \mathrm{meV}$. 


\section{Supplemental references:}

[1] K. Synoradzki, K. Ciesielski, I. Veremchuk, H. Borrmann, P. Skokowski, D. Szymański, Y. Grin, and D. Kaczorowski, Materials. 12, 1723 (2019).

[2] Y. Nakajima, R. Hu, K. Kirshenbaum, A. Hughes, P. Syers, X. Wang, K. Wang, R. Wang, S. R. Saha, D. Pratt, J. W. Lynn, and J. Paglione, Sci. Adv. 1, e1500242 (2015).

[3] O. Pavlosiuk, D. Kaczorowski, and P. Wiśniewski, Sci. Rep. 5, 9158 (2015).

[4] O. Pavlosiuk, D. Kaczorowski, X. Fabreges, A. Gukasov, and P. Wiśniewski, Sci. Rep. 6, 18797 (2016).

[5] E. D. Mun, S. L. Bud'ko, C. Martin, H. Kim, M. A. Tanatar, J.-H. Park, T. Murphy, G. M. Schmiedeshoff, N. Dilley, R. Prozorov, and P. C. Canfield, Phys. Rev. B 87, 075120 (2013).

[6] O. Pavlosiuk, D. Kaczorowski, and P. Wiśniewski, Phys. Rev. B 94, 035130 (2016).

[7] N. P. Butch, P. Syers, K. Kirshenbaum, A. P. Hope, and J. Paglione, Phys. Rev. B 84, 220504 (2011).

[8] Z. Hou, Y. Wang, E. Liu, H. Zhang, W. Wang, and G. Wu, Appl. Phys. Lett. 107, 202103 (2015).

[9] M. Hirschberger, S. Kushwaha, Z. Wang, Q. Gibson, S. Liang, C. A. Belvin, B. A. Bernevig, R. J. Cava, and N. P. Ong, Nat. Mater. 15, 1161 (2016).

[10] Y. Wu, N. H. Jo, M. Ochi, L. Huang, D. Mou, S. L. Bud'ko, P. C. Canfield, N. Trivedi, R. Arita, and A. Kaminski, Phys. Rev. Lett. 115, 166602 (2015).

[11] Y. Zhang, C. Wang, L. Yu, G. Liu, A. Liang, J. Huang, S. Nie, X. Sun, Y. Zhang, B. Shen, J. Liu, H. Weng, L. Zhao, G. Chen, X. Jia, C. Hu, Y. Ding, W. Zhao, Q. Gao, C. Li, S. He, L. Zhao, F. Zhang, S. Zhang, F. Yang, Z. Wang, Q. Peng, X. Dai, Z. Fang, Z. Xu, C. Chen, and X. J. Zhou, Nat. Commun. 8, 15512 (2017).

[12] H. Chi, C. Zhang, G. Gu, D.E. Kharzeev, X. Dai, and Q. Li, New J. Phys. 19, 015005 (2017).

[13] H.-J. Kim, K.-S. Kim, J.-F. Wang, M. Sasaki, N. Satoh, A. Ohnishi, M. Kitaura, M. Yang, and L. Li, Phys. Rev. Lett. 111, 246603 (2013).

[14] D. T. Son and B. Z. Spivak, Phys. Rev. B 88, 104412 (2013).

[15] G. Ding, G. Y. Gao, L. Yu, Y. Ni, and K. Yao, J. Appl. Phys. 119, 025105 (2016).

[16] K. Kaur, S. Dhiman, and R. Kumar, Phys. Lett. A 381, 339 (2017). 